\title{
Pair production of Higgs bosons at the LHC in gauged 2HDM
}

\author{
Chuan-Ren Chen, ${ }^{1}$ Yu-Xiang Lin, ${ }^{1}$ Van Que Tran, ${ }^{1}$ and Tzu-Chiang Yuan ${ }^{2}$ \\ ${ }^{1}$ Department of Physics, National Taiwan Normal University, Taipei 116, Taiwan \\ ${ }^{2}$ Institute of Physics, Academia Sinica, Nangang, Taipei 11529, Taiwan
}

(Received 31 October 2018; published 24 April 2019)

\begin{abstract}
Pair production of Higgs bosons at the Large Hadron Collider (LHC) is known to be important for the determination of the Higgs boson self-coupling and as a probe of new physics beyond the Standard Model (SM), especially the existence of new fundamental scalar bosons. In this paper we study in detail the pair production of Higgs bosons at the LHC in a well-motivated model-the gauged two-Higgs-doublet model - in which the two Higgs doublets are properly embedded into a gauged $S U(2)_{H}$ and a dark matter candidate emerges naturally due to the gauge symmetry. Besides the deviations of the Higgs couplings from the SM predictions, the existence of new scalars could significantly enhance the production cross section of Higgs boson pairs at the LHC. However, when we take into account the relic density of dark matter and the null results of direct detection experiments, only a moderate enhancement can be obtained. We also comment on the capability of the LHC to distinguish the Higgs pair signal due to a generic $400 \mathrm{GeV}$ scalar resonance in the $\gamma \gamma b \bar{b}$ final state from the SM prediction, assuming that their Higgs pair production cross sections are the same.
\end{abstract}

DOI: 10.1103/PhysRevD.99.075027

\section{INTRODUCTION}

The year 2018 marked the 50th anniversary of the Standard Model (SM) of particle physics since its inception in 1967 [1]. The Higgs boson, the long-sought last particle in the SM, was ultimately discovered in 2012 at the Large Hadron Collider (LHC) with a relatively light mass of $125 \mathrm{GeV}$. Since then many efforts have been made to determine whether the observed Higgs boson is indeed the one predicted by the SM, in which the simple Higgs mechanism is used for spontaneous symmetry breaking, as opposed to other alternatives involving strongly interacting dynamics like composite-Higgs or technicolor models. In particular, all of the measurements related to the Higgs' couplings with the SM gauge bosons have been analyzed in detail and nothing has been detected outside the norm. Recently, six years after its discovery, the decay of the Higgs boson into its dominant mode- a pair of bottom quarks - was finally observed at a significance above 5 standard deviations and also in line with the SM expectation $[2,3]$.

Despite its many triumphs, the SM leaves us with many questions to be answered, such as the origin of flavor, the hierarchy/fine-tuning problem, and so on. In the Higgs

Published by the American Physical Society under the terms of the Creative Commons Attribution 4.0 International license. Further distribution of this work must maintain attribution to the author(s) and the published article's title, journal citation, and DOI. Funded by SCOAP. sector, the self-coupling of the Higgs boson and the related issue of the shape of the Higgs potential need to be determined. Moreover, the observations of neutrino oscillations, the relic density of dark matter (DM), matterantimatter asymmetry, etc. imply that there must be new physics beyond the SM.

Many new models have been proposed in the literature to address the above issues, and one of the simplest proposals is an extension of the scalar sector of the SM. For example, the general two-Higgs-doublet model (2HDM) is a simple extension [4] that only adds one more Higgs doublet to the SM. One particular type of $2 \mathrm{HDM}$ is the inert Higgsdoublet model (IHDM) [5-9] in which the neutral component of the second Higgs doublet is a DM candidate. The stability of this DM candidate is ensured by imposing a discrete $Z_{2}$ symmetry on the scalar potential of the model. The presence of the dangerous tree-level flavor-changing neutral current in the $2 \mathrm{HDM}$ can also be cured by the $Z_{2}$ symmetry [10].

Recently, a gauged two-Higgs-doublet model (G2HDM) [11] was proposed. Like the 2HDM, two Higgs doublets $H_{1}$ and $H_{2}$ are introduced but they can be rotated into each other by a gauge transformation under a new non-Abelian $S U(2)_{H}$. Thus, they form a doublet $H=\left(H_{1}, H_{2}\right)^{\mathrm{T}}$ under $S U(2)_{H}$. The neutral component of $H_{2}$ is stable under the protection of $S U(2)_{H}$ gauge symmetry and hence can be a DM candidate. The local $S U(2)_{H}$ gauge symmetry also prevents tree-level flavor-changing neutral currents in the Higgs couplings with the SM fermions. We note that previous studies used an additional Abelian $U(1)$ local 
symmetry in the $2 \mathrm{HDM}$ to obtain a dark matter candidate [12] and natural conservation laws for flavor-changing neutral currents $[13,14]$. To make the G2HDM selfconsistent, however, more new particles are introduced, including an additional $S U(2)_{H}$ doublet, an $S U(2)_{H}$ triplet, and heavy $S U(2)_{L}$ singlet Dirac fermions. Some phenomenology of the G2HDM at the LHC was explored previously in Refs. [11,15] for Higgs physics and in Ref. [16] for the new gauge bosons. Recently, a detailed study of the theoretical and Higgs phenomenological constraints in the G2HDM was presented in Ref. [17]. In this paper, we focus on Higgs pair production in the G2HDM at the LHC, a well-known process that can play a crucial role in the determination of the Higgs boson self-coupling. A similar study for the case of the 2HDM can be found in Ref. [18].

After electroweak symmetry breaking, the scalar potential of the SM can be written as

$$
V_{\mathrm{SM}}=\frac{m_{h}^{2}}{2} h^{2}+\lambda_{\mathrm{SM}} v h^{3}+\frac{\lambda_{\mathrm{SM}}}{4} h^{4}
$$

where $m_{h}$ is the Higgs boson mass $\sim 125 \mathrm{GeV}$ and $v=$ $246 \mathrm{GeV}$ is the Higgs vacuum expectation value (VEV). In the SM, $\lambda_{\mathrm{SM}}=\frac{m_{h}^{2}}{2 v^{2}}$ and has a numerical value of 0.13 . While the first term in the above Higgs potential (or Higgs boson mass term) has been measured at the LHC, the second and third terms (Higgs boson self-coupling terms) have not yet been measured. These trilinear and quartic self-couplings of the Higgs are key parameters for the reconstruction of the Higgs potential, which can tell us how electroweak symmetry breaking really happens and whether the Higgs sector agrees with the SM. While more precise measurements of single Higgs production as well as other associated production channels at the LHC are important to pin down the detailed properties of Higgs couplings in the SM, ${ }^{1}$ Higgs pair production is quite unique in the sense that it can allow us to directly search for the trilinear Higgs selfcoupling in Eq. (1). It has been shown that the Higgs trilinear coupling can be determined with about 50\% accuracy at the $14 \mathrm{TeV}$ LHC with $3000 \mathrm{fb}^{-1}$ of data $[21,22]$. To probe for the quartic self-coupling term in Eq. (1), one would need to study triple Higgs production which might not be accessible at the LHC due to its minuscule cross section.

The production of Higgs pairs in the G2HDM will differ from the SM prediction in a number of ways:

(1) Modified Yukawa couplings.

(2) Modified trilinear Higgs self-coupling.

\footnotetext{
${ }^{1}$ Actually, single Higgs boson production at the LHC can probe the trilinear Higgs self-coupling indirectly via the electroweak one-loop correction [19,20]. With $3000 \mathrm{fb}^{-1}$ luminosity, it was shown that the deviation of single Higgs boson production data could reach the $2 \sigma$ level if the Higgs trilinear coupling is about 9 times larger or about -4 times smaller than its SM value.
}

(3) Presence of new colored particles which can flow inside the triangle and box loops.

(4) Presence of new heavy scalars which can decay into a pair of $125 \mathrm{GeV}$ Higgs bosons via new trilinear scalar couplings. This has important implications for the resonant Higgs boson pair production cross section.

We will include all of these new features in our analysis of Higgs pair production in the G2HDM at the LHC.

This paper is organized as follows. In Sec. II we first review the setup of the G2HDM with a special focus on the scalar sector, i.e., the symmetry breaking of the scalar potential and the resulting scalar spectra. In Sec. III we review the theoretical and phenomenological constraints imposed on this model that have been studied recently in Ref. [17]. In Sec. IV we discuss the Higgs pair production cross section in the G2HDM and present the numerical results. In Sec. V, we present a detailed Monte Carlo simulation study for the two final states of $\gamma \gamma b \bar{b}$ and $b \bar{b} b \bar{b}$ from double Higgs production. Finally, we conclude our study in Sec. VI.

\section{THE MODEL}

To make this paper somewhat self-contained, we will briefly review the G2HDM in this section and refer our readers to Refs. $[11,17]$ for more details. The gauge group of the G2HDM is $\mathcal{G}_{\mathrm{G} 2 \mathrm{HDM}}=\mathcal{G}_{\mathrm{SM}} \times S U(2)_{H} \times U(1)_{X}$, where $\mathcal{G}_{\mathrm{SM}}=S U(3)_{C} \times S U(2)_{L} \times U(1)_{Y}$ is the SM gauge group. The scalar sector includes not only the two $S U(2)_{L}$ Higgs doublets $H_{1}, H_{2}$ which form a doublet $H=$ $\left(H_{1}, H_{2}\right)^{\mathrm{T}}$ under the $S U(2)_{H}$ gauge group, but also a triplet $\Delta_{H}$ and a doublet $\Phi_{H}$ of this new gauge group. Note that both $\Delta_{H}$ and $\Phi_{H}$ are singlets under $\mathcal{G}_{\mathrm{SM}}$. Furthermore, $H$ and $\Phi_{H}$ are assigned to carry an additional $U(1)_{X}$ charge. $^{2}$ For the fermion sector, the SM left-handed $S U(2)_{L}$ doublets $Q_{L}, L_{L}$ are singlets under $S U(2)_{H}$, while the $\mathrm{SM}$ right-handed $S U(2)_{L}$ singlets $u_{R}, d_{R}, \nu_{R}, e_{R}^{3}$ are now paired up with new right-handed singlets $u_{R}^{H}, d_{R}^{H}, \nu_{R}^{H}, e_{R}^{H}$ to form doublets $U_{R}, D_{R}, N_{R}, E_{R}$ under $S U(2)_{H}$. Furthermore, for anomaly cancellations, new heavy left-handed fermions $u_{L}^{H}, d_{L}^{H}, \nu_{L}^{H}, e_{L}^{H}$ are needed, which are singlets under both $S U(2)_{L}$ and $S U(2)_{H}$ gauge groups. In Table I, we summarize the matter content and their quantum number assignments in the G2HDM.

\section{A. Higgs potential}

The most general renormalizable Higgs potential invariant under both $S U(2)_{L} \times U(1)_{Y}$ and $S U(2)_{H} \times U(1)_{X}$ is given by [17]

\footnotetext{
${ }^{2}$ This additional $U(1)_{X}$ was introduced to simplify the scalar potential and to avoid possible mixings between the SM fermions and new heavy fermions in the G2HDM.

${ }^{3}$ We also add right-handed neutrinos $\nu_{R} \mathrm{~s}$ to the SM.
} 
TABLE I. Matter content and their quantum number assignments in the G2HDM.

\begin{tabular}{lccccc}
\hline \hline Matter Fields & $S U(3)_{C}$ & $S U(2)_{L}$ & $U(1)_{Y}$ & $S U(2)_{H}$ & $U(1)_{X}$ \\
\hline$H=\left(H_{1} H_{2}\right)^{\mathrm{T}}$ & 1 & 2 & $1 / 2$ & 2 & 1 \\
$\Delta_{H}$ & 1 & 1 & 0 & 3 & 0 \\
$\Phi_{H}$ & 1 & 1 & 0 & 2 & 1 \\
$Q_{L}=\left(u_{L} d_{L}\right)^{\mathrm{T}}$ & 3 & 2 & $1 / 6$ & 1 & 0 \\
$U_{R}=\left(u_{R} u_{R}^{H}\right)^{\mathrm{T}}$ & 3 & 1 & $2 / 3$ & 2 & 1 \\
$D_{R}=\left(d_{R}^{H} d_{R}\right)^{\mathrm{T}}$ & 3 & 1 & $-1 / 3$ & 2 & -1 \\
$u_{L}^{H}$ & 3 & 1 & $2 / 3$ & 1 & 0 \\
$d_{L}^{H}$ & 3 & 1 & $-1 / 3$ & 1 & 0 \\
$L_{L}=\left(\nu_{L} e_{L}\right)^{\mathrm{T}}$ & 1 & 2 & $-1 / 2$ & 1 & 0 \\
$N_{R}=\left(\nu_{R} \nu_{R}^{H}\right)^{\mathrm{T}}$ & 1 & 1 & 0 & 2 & 1 \\
$E_{R}=\left(e_{R}^{H} e_{R}\right)^{\mathrm{T}}$ & 1 & 1 & -1 & 2 & -1 \\
$\nu_{L}^{H}$ & 1 & 1 & 0 & 1 & 0 \\
$e_{L}^{H}$ & 1 & 1 & -1 & 1 & 0 \\
\hline \hline
\end{tabular}

$V_{T}=V(H)+V\left(\Phi_{H}\right)+V\left(\Delta_{H}\right)+V_{\text {mix }}\left(H, \Delta_{H}, \Phi_{H}\right)$,

where

$$
\begin{gathered}
V(H)=\mu_{H}^{2}\left(H_{1}^{\dagger} H_{1}+H_{2}^{\dagger} H_{2}\right)+\lambda_{H}\left(H_{1}^{\dagger} H_{1}+H_{2}^{\dagger} H_{2}\right)^{2} \\
+\lambda_{H}^{\prime}\left(-H_{1}^{\dagger} H_{1} H_{2}^{\dagger} H_{2}+H_{1}^{\dagger} H_{2} H_{2}^{\dagger} H_{1}\right), \\
V\left(\Phi_{H}\right)=\mu_{\Phi}^{2} \Phi_{H}^{\dagger} \Phi_{H}+\lambda_{\Phi}\left(\Phi_{H}^{\dagger} \Phi_{H}\right)^{2}, \\
V\left(\Delta_{H}\right)=-\mu_{\Delta}^{2} \operatorname{Tr}\left(\Delta_{H}^{2}\right)+\lambda_{\Delta}\left(\operatorname{Tr}\left(\Delta_{H}^{2}\right)\right)^{2},
\end{gathered}
$$

with

$$
\begin{aligned}
& \Delta_{H}=\left(\begin{array}{cc}
\Delta_{3} / 2 & \Delta_{p} / \sqrt{2} \\
\Delta_{m} / \sqrt{2} & -\Delta_{3} / 2
\end{array}\right)=\Delta_{H}^{\dagger} \text { with } \\
& \Delta_{m}=\left(\Delta_{p}\right)^{*} \text { and } \quad\left(\Delta_{3}\right)^{*}=\Delta_{3},
\end{aligned}
$$

and the last term

$$
\begin{aligned}
V_{\text {mix }}( & \left.H, \Delta_{H}, \Phi_{H}\right) \\
= & +M_{H \Delta}\left(H^{\dagger} \Delta_{H} H\right)-M_{\Phi \Delta}\left(\Phi_{H}^{\dagger} \Delta_{H} \Phi_{H}\right) \\
& +\lambda_{H \Phi}\left(H^{\dagger} H\right)\left(\Phi_{H}^{\dagger} \Phi_{H}\right)+\lambda_{H \Phi}^{\prime}\left(H^{\dagger} \Phi_{H}\right)\left(\Phi_{H}^{\dagger} H\right) \\
& +\lambda_{H \Delta}\left(H^{\dagger} H\right) \operatorname{Tr}\left(\Delta_{H}^{2}\right)+\lambda_{\Phi \Delta}\left(\Phi_{H}^{\dagger} \Phi_{H}\right) \operatorname{Tr}\left(\Delta_{H}^{2}\right) .
\end{aligned}
$$

We note that the scalar potential in the G2HDM is $C P$ conserving due to the fact that all terms in $V(H), V\left(\Phi_{H}\right)$, $V\left(\Delta_{H}\right)$, and $V_{\text {mix }}\left(H, \Delta_{H}, \Phi_{H}\right)$ are Hermitian, implying that all of the coefficients are necessarily real.

\section{B. Spontaneous symmetry breaking and scalar mass spectrum}

\section{Spontaneous symmetry breaking}

To facilitate spontaneous electroweak symmetry breaking, we shift the scalar fields as follows:

$$
\begin{aligned}
H_{1}=\left(\begin{array}{c}
G^{+} \\
\frac{v+h}{\sqrt{2}}+i \frac{G^{0}}{\sqrt{2}}
\end{array}\right), & H_{2}=\left(\begin{array}{c}
H^{+} \\
H_{2}^{0}
\end{array}\right), \\
\Phi_{H}=\left(\begin{array}{c}
G_{H}^{p} \\
\frac{v_{\Phi}+\phi_{2}}{\sqrt{2}}+i \frac{G_{H}^{0}}{\sqrt{2}}
\end{array}\right), & \Delta_{H}=\left(\begin{array}{cc}
\frac{-v_{\Delta}+\delta_{3}}{2} & \frac{1}{\sqrt{2}} \Delta_{p} \\
\frac{1}{\sqrt{2}} \Delta_{m} & \frac{v_{\Delta}-\delta_{3}}{2}
\end{array}\right),
\end{aligned}
$$

where $v, v_{\Phi}$, and $v_{\Delta}$ are the VEVs to be determined by minimization of the potential. The set $\Psi_{G} \equiv$ $\left\{G^{0}, G^{+}, G_{H}^{0}, G_{H}^{p}\right\}$ are Goldstone bosons, while the rest are physical fields. Inserting Eq. (8) into the potential $V_{T}$ given by Eq. (2) and keeping only those terms involving the VEVs leads us to

$$
\begin{aligned}
V_{T}\left(v, v_{\Delta}, v_{\Phi}\right)= & \frac{1}{4}\left[\lambda_{H} v^{4}+\lambda_{\Phi} v_{\Phi}^{4}+\lambda_{\Delta} v_{\Delta}^{4}\right. \\
& +2\left(\mu_{H}^{2} v^{2}+\mu_{\Phi}^{2} v_{\Phi}^{2}-\mu_{\Delta}^{2} v_{\Delta}^{2}\right) \\
& -\left(M_{H \Delta} v^{2}+M_{\Phi \Delta} v_{\Phi}^{2}\right) v_{\Delta}+\lambda_{H \Phi} v^{2} v_{\Phi}^{2} \\
& \left.+\lambda_{H \Delta} v^{2} v_{\Delta}^{2}+\lambda_{\Phi \Delta} v_{\Phi}^{2} v_{\Delta}^{2}\right] .
\end{aligned}
$$

To determine the ground state of the model, we take the derivatives of Eq. (9) with respect to $v, v_{\Phi}$, and $v_{\Delta}$ and set them to zero. We can then obtain the following equations satisfied by the VEVs for the minimum energy configuration:

$$
\begin{gathered}
v \cdot\left(2 \lambda_{H} v^{2}+2 \mu_{H}^{2}-M_{H \Delta} v_{\Delta}+\lambda_{H \Phi} v_{\Phi}^{2}+\lambda_{H \Delta} v_{\Delta}^{2}\right)=0, \\
v_{\Phi} \cdot\left(2 \lambda_{\Phi} v_{\Phi}^{2}+2 \mu_{\Phi}^{2}-M_{\Phi \Delta} v_{\Delta}+\lambda_{H \Phi} v^{2}+\lambda_{\Phi \Delta} v_{\Delta}^{2}\right)=0,
\end{gathered}
$$

$$
\begin{gathered}
4 \lambda_{\Delta} v_{\Delta}^{3}-4 \mu_{\Delta}^{2} v_{\Delta}-M_{H \Delta} v^{2}-M_{\Phi \Delta} v_{\Phi}^{2} \\
+2 v_{\Delta}\left(\lambda_{H \Delta} v^{2}+\lambda_{\Phi \Delta} v_{\Phi}^{2}\right)=0 .
\end{gathered}
$$

By solving this set of coupled algebraic equations, one can get nontrivial solutions for all of the VEVs $\left(v, v_{\Phi}\right.$, and $\left.v_{\Delta}\right)$ in terms of the fundamental parameters in the potential [17].

\section{Scalar mass spectrum}

After the electroweak symmetry is broken, we obtain three diagonal blocks in the scalar mass matrix. The first $3 \times 3$ block in the basis of $S=\left\{h, \phi_{2}, \delta_{3}\right\}$ is given by 


$$
\mathcal{M}_{H}^{2}=\left(\begin{array}{ccc}
2 \lambda_{H} v^{2} & \lambda_{H \Phi} v v_{\Phi} & \frac{v}{2}\left(M_{H \Delta}-2 \lambda_{H \Delta} v_{\Delta}\right) \\
\lambda_{H \Phi} v v_{\Phi} & 2 \lambda_{\Phi} v_{\Phi}^{2} & \frac{v_{\Phi}}{2}\left(M_{\Phi \Delta}-2 \lambda_{\Phi \Delta} v_{\Delta}\right) \\
\frac{v}{2}\left(M_{H \Delta}-2 \lambda_{H \Delta} v_{\Delta}\right) & \frac{v_{\Phi}}{2}\left(M_{\Phi \Delta}-2 \lambda_{\Phi \Delta} v_{\Delta}\right) & \frac{1}{4 v_{\Delta}}\left(8 \lambda_{\Delta} v_{\Delta}^{3}+M_{H \Delta} v^{2}+M_{\Phi \Delta} v_{\Phi}^{2}\right)
\end{array}\right)
$$

This matrix can be diagonalized by an orthogonal matrix $O^{H}$,

$$
\left(O^{H}\right)^{\mathrm{T}} \cdot \mathcal{M}_{H}^{2} \cdot O^{H}=\operatorname{Diag}\left(m_{h_{1}}^{2}, m_{h_{2}}^{2}, m_{h_{3}}^{2}\right) .
$$

The lightest eigenvalue $m_{h_{1}}$ is the mass of $h_{1}$, which is identified as the $125 \mathrm{GeV}$ Higgs boson observed at the LHC, while $m_{h_{2}}$ and $m_{h_{3}}$ are the masses of the heavier
Higgses $h_{2}$ and $h_{3}$, respectively. These physical Higgs bosons $h_{i}(i=1,2,3)$ are a mixture of the three components of $S: h_{i}=O_{j i}^{H} S_{j}$. Thus, the SM-like Higgs boson in this model is a linear combination of the neutral components of the two $S U(2)$ doublets $H_{1}$ and $\Phi_{H}$ and the real component of the $S U(2)_{H}$ triplet $\Delta_{H}$.

The second block is also $3 \times 3$. In the basis of $G=\left\{G_{H}^{p}, H_{2}^{0 *}, \Delta_{p}\right\}$, it is given by

$$
\mathcal{M}_{D}^{2}=\left(\begin{array}{ccc}
M_{\Phi \Delta} v_{\Delta}+\frac{1}{2} \lambda_{H \Phi}^{\prime} v^{2} & \frac{1}{2} \lambda_{H \Phi}^{\prime} v v_{\Phi} & -\frac{1}{2} M_{\Phi \Delta} v_{\Phi} \\
\frac{1}{2} \lambda_{H \Phi}^{\prime} v v_{\Phi} & M_{H \Delta} v_{\Delta}+\frac{1}{2} \lambda_{H \Phi}^{\prime} v_{\Phi}^{2} & \frac{1}{2} M_{H \Delta} v \\
-\frac{1}{2} M_{\Phi \Delta} v_{\Phi} & \frac{1}{2} M_{H \Delta} v & \frac{1}{4 v_{\Delta}}\left(M_{H \Delta} v^{2}+M_{\Phi \Delta} v_{\Phi}^{2}\right)
\end{array}\right)
$$

This matrix can also be diagonalized by an orthogonal matrix $O^{D}$,

$$
\left(O^{D}\right)^{\mathrm{T}} \cdot \mathcal{M}_{D}^{2} \cdot O^{D}=\operatorname{Diag}\left(m_{\tilde{G}^{p}}^{2}, m_{D}^{2}, m_{\Delta}^{2}\right) .
$$

One eigenvalue of Eq. (15) is zero (i.e., $m_{\widetilde{G}^{p}}=0$ ) and identified as the unphysical Goldstone boson $\tilde{G}^{p} \cdot m_{D}$ and $m_{\tilde{\Delta}}\left(m_{D}<m_{\tilde{\Delta}}\right)$ are the masses of the two physical fields $D$ and $\tilde{\Delta}$, respectively. $D$ could be a DM candidate in the G2HDM.

The final block is a $4 \times 4$ diagonal matrix with the following entries:

$$
\begin{gathered}
m_{H^{ \pm}}^{2}=-\frac{1}{2} \lambda_{H}^{\prime} v^{2}+\frac{1}{2} \lambda_{H \Phi}^{\prime} v_{\Phi}^{2}+M_{H \Delta} v_{\Delta}, \\
m_{G^{ \pm}}^{2}=m_{G^{0}}^{2}=m_{G_{H}^{0}}^{2}=0,
\end{gathered}
$$

where $m_{H^{ \pm}}$is the mass of the physical charged Higgs $H^{ \pm}$, and $m_{G^{ \pm}}, m_{G^{0}}$, and $m_{G_{H}^{0}}$ are the masses of the four Goldstone boson fields $G^{ \pm}, G^{0}$, and $G_{H}^{0}$, respectively. Note that we have used the minimization conditions (10), (11), and (12) to simplify various matrix elements of the above mass matrices. The six Goldstone particles $G^{ \pm}, G^{0}$, $G_{H}^{0}$, and $\tilde{G}^{p, m}$ will be absorbed by the longitudinal components of the massive gauge bosons $W^{ \pm}, Z, Z^{\prime}$, and $W^{\prime(p, m)}$ after electroweak symmetry breaking. For details about the gauge boson masses, we refer our readers to Refs. [11,17].

\section{CONSTRAINTS}

Before embarking on our numerical study, we summarize in this section the allowed parameter space of the scalar sector of the model.

The constraints were obtained in Ref. [17], through the requirements of vacuum stability (VS), perturbative unitarity (PU), and Higgs physics (HP), the latter of which includes the Higgs boson mass of $125 \mathrm{GeV}$ and signal strengths of Higgs boson decays into a diphoton and $\tau^{+} \tau^{-}$ measured at the LHC. It should be noted that, in the numerical study of Ref. [17], the two parameters $M_{H \Delta}$ and $M_{\Phi \Delta}$ in $V_{\text {mix }}$ were set to vary in the range $[-1,1] \mathrm{TeV}$ and $v_{\Delta} \in[0.5,20] \mathrm{TeV}$, while $v$ and $v_{\Phi}$ were fixed to be $246 \mathrm{GeV}$ and $10 \mathrm{TeV}$, respectively.

We show a summary of the allowed regions of parameter space in Fig. 1 obtained in Ref. [17]. The upper red triangular block corresponds to (VS + PU) constraints, while the lower magenta triangular block corresponds to the (VS + PU + HP) constraints. The diagonal panels indicate the allowed ranges of the eight couplings $\lambda_{H, \Phi, \Delta}, \lambda_{H}^{\prime}$, and $\lambda_{H \Phi, H \Delta, \Phi \Delta}, \lambda_{H \Phi}^{\prime}$ under the combined constraints of (VS + PU + HP). It turns out that among the eight $\lambda$ parameters, only two of them $\left(\lambda_{H}\right.$ and $\left.\lambda_{H \Phi}\right)$ are significantly constrained by (VS $+\mathrm{PU}+\mathrm{HP})$. In particular, $\lambda_{H}$ (the Higgs self-coupling in the G2HDM) no longer has the fixed SM value of 0.13 but rather is allowed to vary in the range $(0.13,4.00)$. We note that some of the parameters such as $M_{H \Delta}, M_{\Phi \Delta}$, and the VEVs are only constrained by HP, and not by (VS + PU).

We should also emphasize that the VS and PU constraints that we consider in this work are tree-level results. Higher-order corrections like renormalization group 


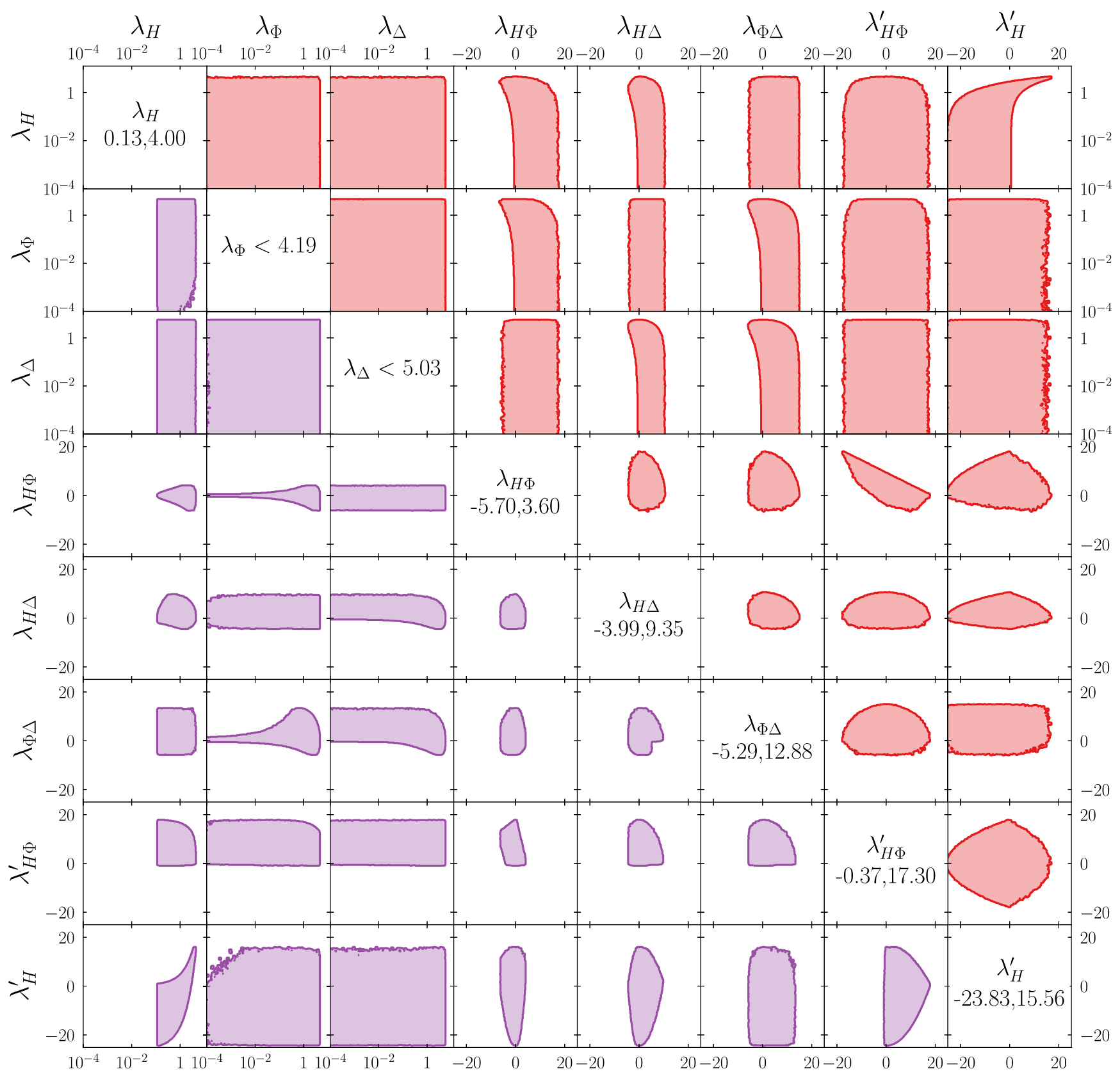

FIG. 1. Summary of the parameter space allowed by the theoretical and phenomenological constraints. The red regions show the results from the theoretical constraints (VS + PU). The magenta regions are constrained by Higgs physics as well as the theoretical constraints (VS + PU + HP). Diagonal blocks indicate the allowed ranges for the scalar couplings from (VS+PU+HP) constraints. This figure is taken from Ref. [17].

evolution effects in the quartic scalar couplings are important. However, they are beyond the scope of this work and we leave them to future studies.

\section{HIGGS BOSON PAIR PRODUCTION IN THE G2HDM AT THE LHC}

In the SM, a pair of Higgs bosons can be produced via two channels at the LHC: a triangle loop diagram with a Higgs boson as the mediator, and a box loop diagram.
However, the small Higgs boson pair production rate, which is roughly a thousand times smaller than single Higgs boson production at the $14 \mathrm{TeV}$ LHC [23-33], makes the measurement very challenging. We quote here a recent combined observed (expected) limit by ATLAS [34] on the nonresonant Higgs boson pair cross section of $0.22 \mathrm{pb}(0.35 \mathrm{pb})$ at the $95 \%$ confidence level, which corresponds to 6.7 (10.4) times the predicted SM cross section. Clearly, these limits are rather loose and not very useful for deducing meaningful constraints in any model. 


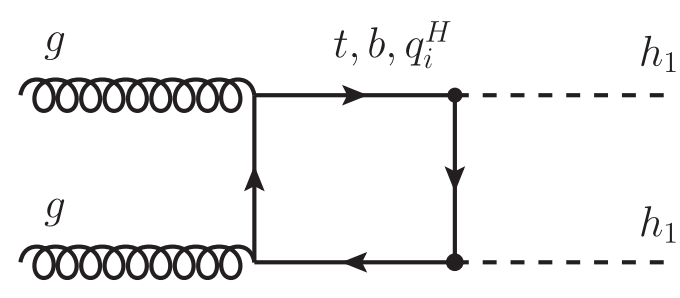

(a)

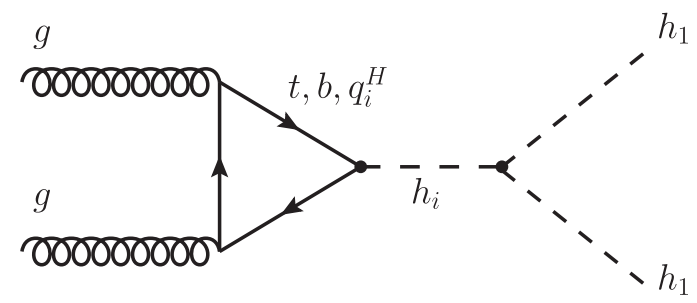

(b)

FIG. 2. Box (a) and triangle (b) Feynman diagrams for the production of a pair of 125 GeV Higgs bosons in the G2HDM. Note that $q_{i}^{H}=u^{H}, d^{H}, c^{H}, s^{H}, t^{H}, b^{H}$ and $h_{i}=h_{1}, h_{2}, h_{3}$.

Nevertheless, in the G2HDM the Higgs boson pair production rate can be significantly enhanced since more diagrams contribute, including the new heavy quarks $q^{H}$ in the loop and heavy scalars $h_{2}, h_{3}$ as mediators. In addition, the $125 \mathrm{GeV}$ SM-like Higgs boson $h_{1}$ is a mixture of $h, \phi_{2}$, and $\delta_{3}$, and this mixing effect will modify both the quark Yukawa couplings and the trilinear Higgs self-coupling. The Feynman diagrams for the production of a pair of $h_{1}$ 's in the G2HDM are shown in Fig. 2. In the SM the top-quark loop contribution is dominant, while the bottom-quark contribution is negative and negligible. The contributions between the box and triangle diagrams also interfere destructively in the SM. The relevant couplings for the production of a pair of $h_{1}$ 's in the G2HDM are as follows:

$$
\begin{aligned}
g_{q q h_{i}} & =O_{1 i}^{H} \frac{m_{q}}{v}, \\
g_{q^{H} q^{H} h_{i}} & =O_{2 i}^{H} \frac{m_{q^{H}}}{v_{\Phi}},
\end{aligned}
$$

$$
\begin{aligned}
g_{h_{2} h_{1} h_{1}}= & 6\left(\lambda_{H} v\left(O_{11}^{H}\right)^{2} O_{12}^{H}+\lambda_{\Phi} v_{\Phi}\left(O_{21}^{H}\right)^{2} O_{22}^{H}-\lambda_{\Delta} v_{\Delta}\left(O_{31}^{H}\right)^{2} O_{32}^{H}\right)+\frac{1}{2} M_{H \Delta} O_{11}^{H}\left(O_{11}^{H} O_{32}^{H}+2 O_{12}^{H} O_{31}^{H}\right) \\
& +\frac{1}{2} M_{\Phi \Delta} O_{21}^{H}\left(O_{21}^{H} O_{32}^{H}+2 O_{22}^{H} O_{31}^{H}\right)+\lambda_{H \Delta}\left[v\left(\left(O_{31}^{H}\right)^{2} O_{12}^{H}+2 O_{11}^{H} O_{31}^{H} O_{32}^{H}\right)-v_{\Delta}\left(\left(O_{11}^{H}\right)^{2} O_{32}^{H}+2 O_{11}^{H} O_{12}^{H} O_{31}^{H}\right)\right] \\
& +\lambda_{\Phi \Delta}\left[v_{\Phi}\left(\left(O_{31}^{H}\right)^{2} O_{22}^{H}+2 O_{21}^{H} O_{31}^{H} O_{32}^{H}\right)-v_{\Delta}\left(\left(O_{21}^{H}\right)^{2} O_{32}^{H}+2 O_{21}^{H} O_{22}^{H} O_{31}^{H}\right)\right] \\
& +\lambda_{H \Phi}\left[v\left(\left(O_{21}^{H}\right)^{2} O_{12}^{H}+2 O_{11}^{H} O_{21}^{H} O_{22}^{H}\right)+v_{\Phi}\left(O_{11}^{H}\left(O_{11}^{H} O_{22}^{H}+2 O_{12}^{H} O_{21}^{H}\right)\right)\right]
\end{aligned}
$$

where $g_{q q h_{i}}, g_{q^{H} q^{H} h_{i}}, g_{h_{1} h_{1} h_{1}}$, and $g_{h_{2} h_{1} h_{1}}$ are the SM quark Yukawa couplings, new heavy quark Yukawa couplings, trilinear $h_{1}$ self-coupling, and coupling between the heavier scalar $h_{2}$ and two $h_{1}$ 's, respectively. One can see that the SM quark Yukawa couplings $g_{q q h_{1}}$ are now smaller by a factor of the mixing element $O_{11}^{H}$ as compared to the SM values. Since the modifications of the SM Yukawa couplings are always suppressed by the same factor of $O_{11}^{H}$ for the top and bottom quarks, the role of the bottom quark is never significant for double $h_{1}$ production in the G2HDM. Furthermore, the scalar self-couplings $g_{h_{1} h_{1} h_{1}}$ and $g_{h_{2} h_{1} h_{1}}$ in the G2HDM are comprised of many new parameters which can affect the double $h_{1}$ production. In what follows, we will ignore the heaviest scalar mediator $h_{3}$ in our analysis due to its negligible contribution to the double $h_{1}$ production cross section from Fig. 2(b).
The differential cross section for double $h_{1}$ production from gluon fusion in the G2HDM can be straightforwardly translated from the SM formulas [33],

$$
\begin{aligned}
& \frac{d \hat{\sigma}\left(g g \rightarrow h_{1} h_{1}\right)}{d \hat{t}} \\
& =\frac{G_{F}^{2} \alpha_{s}^{2}}{512(2 \pi)^{3}} \times\left\{\mid \sum_{f=q, q^{H}} \sum_{i=1}^{2} \kappa_{f f h_{i}} g_{h_{i} h_{1} h_{1}} v D_{h_{i}}(\hat{s}) F_{\Delta}\left(\hat{s}, \tau_{f i}\right)\right. \\
& \left.\quad+\left.\sum_{f=q, q^{H}} \kappa_{f f h_{1}}^{2} F_{\square}\left(\hat{s}, \tau_{f i}\right)\right|^{2}+\left|\sum_{f=q, q^{H}} \kappa_{f f h_{1}}^{2} G_{\square}\left(\hat{s}, \tau_{f i}\right)\right|^{2}\right\},
\end{aligned}
$$

where $G_{F}$ and $\alpha_{s}$ are the Fermi constant and strong coupling constant, respectively, 


$$
\begin{gathered}
\kappa_{q q h_{i}}=O_{1 i}^{H}, \\
\kappa_{q^{H} q^{H} h_{i}}=O_{2 i}^{H} \frac{v}{v_{\Phi}}, \\
D_{h_{i}}(\hat{s})=\frac{1}{\left(\hat{s}-m_{h_{i}}^{2}+i m_{h_{i}} \Gamma_{h_{i}}\right)},
\end{gathered}
$$

and $F_{\Delta}\left(\hat{s}, \tau_{f i}\right), F_{\square}\left(\hat{s}, \tau_{f i}\right), G_{\square}\left(\hat{s}, \tau_{f i}\right)$ with $\tau_{f i}=4 m_{f}^{2} / m_{h_{i}}^{2}$ are form factors that can be found in Appendix A.1 of Ref. [33]. For later purposes, we also define $\lambda_{h_{i} h_{1} h_{1}}=$ $g_{h_{i} h_{1} h_{1}} / g_{h h h}^{\mathrm{SM}}$ where $g_{h h h}^{\mathrm{SM}}=6 \lambda_{\mathrm{SM}} v=192 \mathrm{GeV}$.

In this section we present the numerical analysis for the double $h_{1}$ production cross section in the G2HDM at the LHC and compare the results with SM predictions. Monte Carlo studies of the two final states $\gamma \gamma b \bar{b}$ and $b \bar{b} b \bar{b}$ from the $h_{1}$ pair decay will be presented in the next section.

Before we proceed, let us present the setup of the model parameter space used in our analysis. We adopt similar procedures as in the previous work [17]. For the eight $\lambda$ parameters $\lambda_{H, \Phi, \Delta}, \lambda_{H}^{\prime}, \lambda_{H \Phi, H \Delta, \Phi \Delta}, \lambda_{H \Phi}^{\prime}$, we impose their allowed ranges from the theoretical constraints of (VS + PU) as obtained in Ref. [17]. Recall that only these quartic couplings in the scalar potential are relevant for the theoretical constraints of (VS + PU). For the HP constraints, the other four parameters $v_{\Delta}, v_{\Phi}, M_{H \Delta}$, and $M_{\Phi \Delta}$ in the scalar potential are also involved, and they vary in the following ranges:

$$
\begin{gathered}
0.1 \mathrm{GeV}<v_{\Delta}<4 \mathrm{TeV}, \\
30 \mathrm{TeV}<v_{\Phi}<100 \mathrm{TeV}, \\
-3 \mathrm{TeV}<M_{H \Delta}<3 \mathrm{TeV}, \\
0<M_{\Phi \Delta}<15 \mathrm{GeV} .
\end{gathered}
$$

The SM VEV $v$ is fixed at $246 \mathrm{GeV}$. First, we scan all of the parameters in the ranges defined above and require them to pass all of the (VS $+\mathrm{PU}+\mathrm{HP})$ constraints presented in Ref. [17]. The constraints from direct $Z^{\prime}$ resonance searches using the latest ATLAS and CMS $13 \mathrm{TeV}$ results [35-38] have been taken into account in our scan. Furthermore, the dark matter candidate $D$ is set to be heavier than half of the Higgs boson mass so that the invisible mode of $h_{1} \rightarrow D D^{*}$ is not kinematically allowed. For simplicity, the masses of the new heavy fermions $f_{i}^{H}$ are assumed to be degenerate and set to $3 \mathrm{TeV}$. Finally, we focus on the situation in which $m_{h_{2}}>2 m_{h_{1}}$ to allow $h_{2}$ decays on shell into $h_{1} h_{1}$.

In Fig. 3, we show the scatter plots of the ratio of the production cross sections for a pair of $125 \mathrm{GeV}$ Higgs bosons between the G2HDM and SM in the planes $\left(\lambda_{h_{1} h_{1} h_{1}}\right.$,

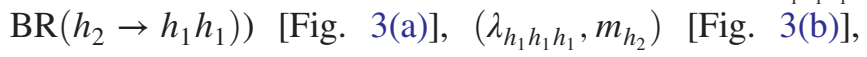

$\left(\lambda_{h_{1} h_{1} h_{1}}, \kappa_{q q h_{1}}\right)$ [Fig. 3(c)], and $\left(\lambda_{h_{1} h_{1} h_{1}}, \kappa_{q q h_{2}}\right)$ [Fig. 3(d)]. The color palette on the right of each of the plots in Fig. 3 indicates the signal strength of the double $h_{1}$ Higgs boson production. From these four plots in Fig. 3, one can see that the trilinear self-coupling of the $125 \mathrm{GeV}$ Higgs boson $\lambda_{h_{1} h_{1} h_{1}}$ in the G2HDM can significantly deviate from its SM value. Even its sign can become negative. From Fig. 3(a), one observes that the branching ratio of the heavier scalar $h_{2}$ decaying into a pair of $h_{1}$ 's can vary from 0 up to $100 \%$. As expected, as $\left|\lambda_{h_{1} h_{1} h_{1}}\right|$ and $\operatorname{BR}\left(h_{2} \rightarrow h_{1} h_{1}\right)$ increase, the triangle diagram becomes the dominant channel and enhances the production cross section. Note that when $\lambda_{h_{1} h_{1} h_{1}}$ becomes negative, there is constructive interference between the box and triangle Feynman diagrams in Fig. 2, whereas in the SM these two diagrams interfere destructively. However, when one of the channels (either the box or triangle Feynman diagram) becomes the dominant contribution to the total production cross section, the interference effect is no longer significant. It is also shown in Fig. 3(b) that for a heavier $h_{2}$ mass the cross section of double $h_{1}$ production will be much smaller. This is also the reason why one can neglect the contribution from the mediator $h_{3}$ since it is much heavier than $h_{2}$. Furthermore, due to the constraints from Higgs physics, the absolute values of the Yukawa couplings of SM quarks with $h_{1}$ cannot deviate too much from their SM values, which is demonstrated in Fig. 3(c), while Fig. 3(d) shows that the Yukawa couplings between the SM quark and $h_{2}$ could be small due to the smallness of the mixing between the $S U(2)_{L}$ doublet scalar $H$ and $S U(2)_{H}$ doublet scalar $\Phi_{H}$, encapsulated by the matrix element $O_{12}^{H}$. The contributions from the new heavy quarks $q^{H}$ in the G2HDM are found to be small because the Yukawa couplings $\kappa_{q^{H} q^{H} h_{i}}$ in Eq. (25) are scaled by the small VEV ratio $v / v_{\Phi}$.

Since a dark matter candidate exists in the G2HDM, we consider further the dark matter constraints from cosmological observations and direct search experiments. We use MicROMEGAs 5.0 [39] to calculate the relic density of the DM candidate and its elastic scattering cross sections with the nucleon. In Fig. 4, we present the scatter plots for the ratio of the production cross sections $\sigma_{g g \rightarrow h_{1} h_{1}} / \sigma_{\mathrm{SM}}$ for a pair of $125 \mathrm{GeV}$ Higgs bosons between the G2HDM and SM in the planes of DM mass $m_{D}$ versus its relic density $\Omega h^{2}$ [Fig. 4(a)] and versus the spin-independent cross section of DM and the nucleon $\sigma_{\text {SI }}^{n}$ [Fig. 4(b)]. The lime (yellow) band corresponds to the $1 \sigma(3 \sigma)$ range of Planck's DM relic density measurement [40]. The orange and black lines represent the upper limits on the spin-independent cross section of DM and the nucleon from the PandaX-II experiment [41] and XENON1T [42], respectively. Imposing the mass of the dark matter candidate $D$ to be the lightest among $\tilde{\Delta}, W^{\prime(p, m)}, H^{ \pm}$, and the new heavy fermions $f_{i}^{H}$ means that $m_{D}$ must be less than $\sim 2.7 \mathrm{TeV}$. In the region $m_{D}>500 \mathrm{GeV}$, there are correlations between 


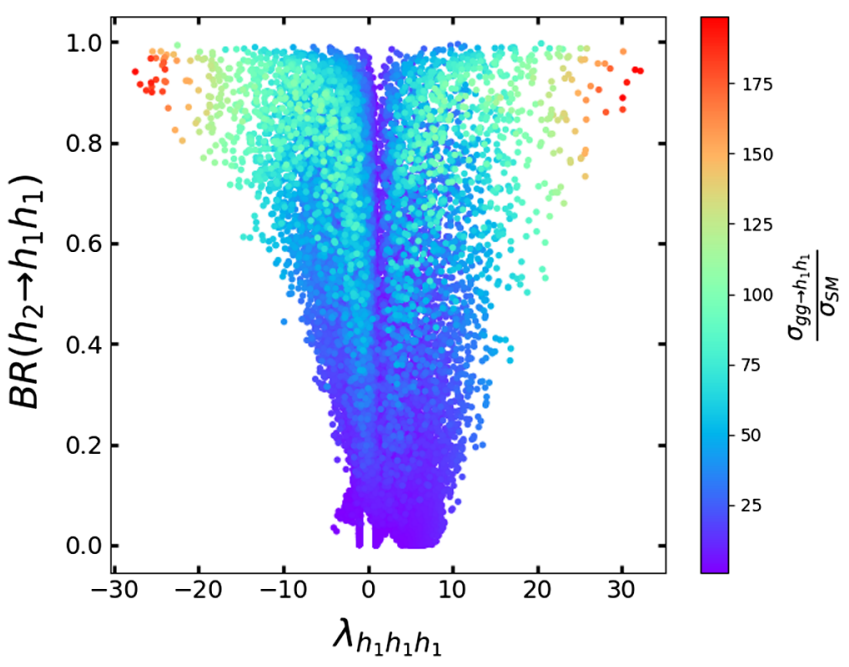

(a)

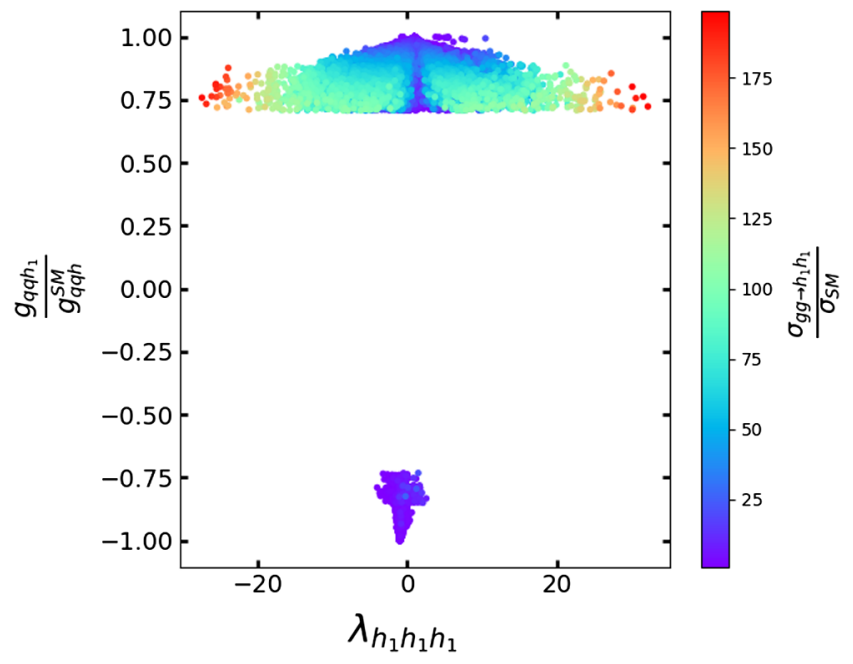

(c)

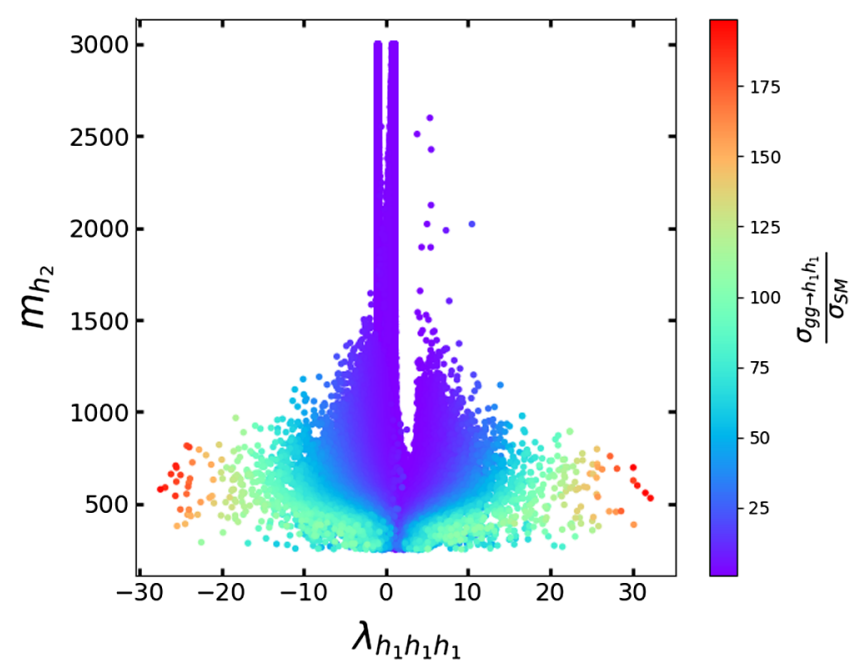

(b)

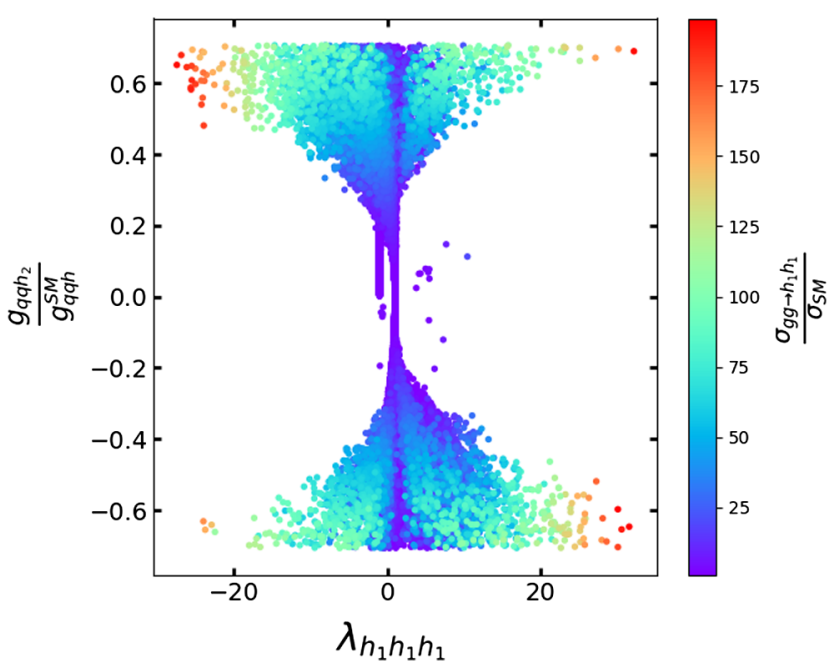

(d)

FIG. 3. Scatter plots of relevant parameters for Higgs boson pair production without the experimental constraints from DM relic density and direct searches. The color palette indicates the ratio of double Higgs boson production cross sections between the G2HDM and SM. Note that $\lambda_{h_{1} h_{1} h_{1}}=g_{h_{1} h_{1} h_{1}} / g_{h h h}^{\mathrm{SM}}$ with $g_{h h h}^{\mathrm{SM}}=6 \lambda_{\mathrm{SM}} v, \kappa_{q q h_{1}}=q_{q q h_{1}} / q_{q q h}^{\mathrm{SM}}$, and $\kappa_{q q h_{2}}=q_{q q h_{2}} / q_{q q h}^{\mathrm{SM}}$ with $q_{q q h}^{\mathrm{SM}}=m_{q} / v$.

the Higgs boson pair production cross section and DM relic density as well as th eDM-nucleon cross section. In particular, the cross section of gluon-gluon fusion to double $h_{1}$ tends to be larger when the DM relic density becomes smaller or the DM-nucleon cross section becomes larger. The first correlation, shown in Fig. 4(a), is due to the fact that $\left|\lambda_{h_{1} h_{1} h_{1}}\right|$ and $\operatorname{BR}\left(h_{2} \rightarrow h_{1} h_{1}\right)$ can control not only Higgs boson pair production at the LHC but also the annihilation cross section of DM into Higgs boson pairs for the relic density calculation. Indeed, when they both become bigger, the DM annihilation process will be dominated by the $D D^{*} \rightarrow h_{i} \rightarrow h_{1} h_{1}$ channel, implying that the DM annihilation cross section becomes larger or the DM relic density becomes smaller. The second correlation, shown in Fig. 4(b), is due to the fact that the DM-nucleon cross section receives about half of its contributions from the one-loop heavy quarks (mainly the top quark) in the triangle diagram which also appear in the double Higgs boson production process.

The DM relic density and direct searches put stringent constraints on the parameter space of the G2HDM. As shown in Fig. 4(a), Planck's relic density measurements constrain the parameter space in a small $3 \sigma$ band, while from Fig. 4(b) one can also see that the DM direct search constraints cut off almost all of the parameter space which significantly enhances the cross section of double Higgs boson production. To be more specific, when both the relic density and direct search constraints are imposed, only about $2 \%$ of the data points survive.

As in Fig. 3, we show in Fig. 5 the scatter plots of the relevant parameters for Higgs boson pair production after taking into account the constraints from DM relic density 


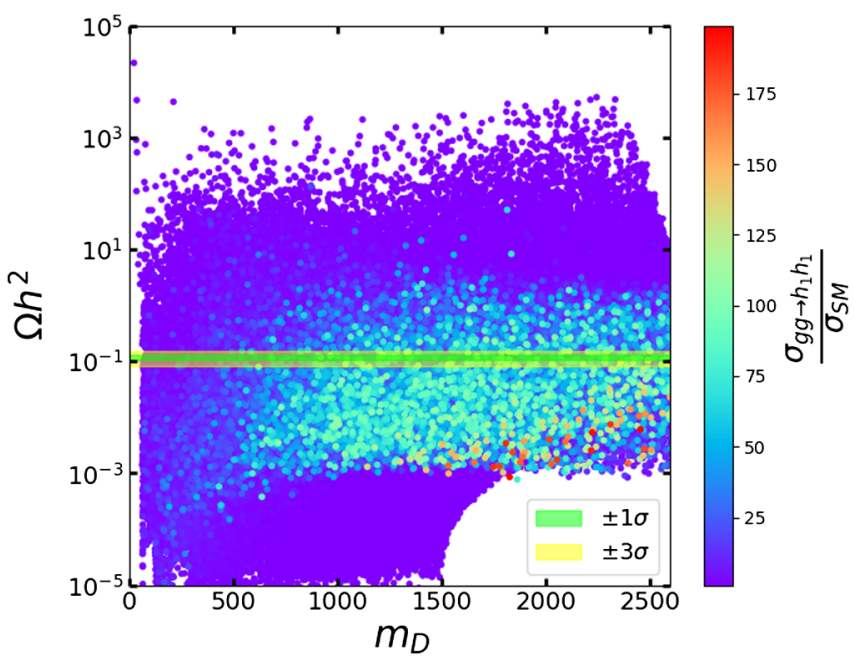

(a)

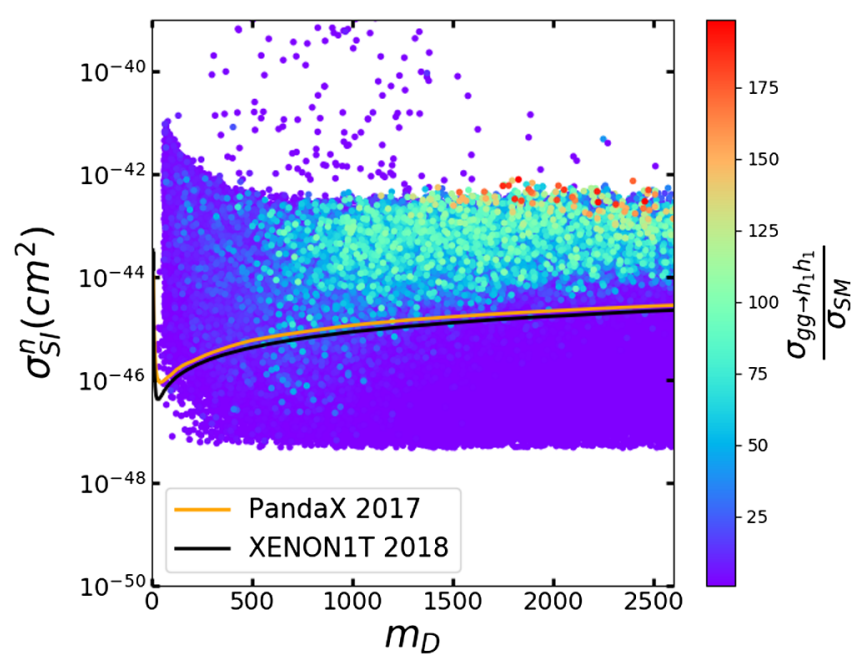

(b)

FIG. 4. Scatter plots for the ratio of the production cross sections for a pair of $125 \mathrm{GeV}$ Higgs bosons between the G2HDM and SM in the planes of DM mass versus its relic density (a) and versus the spin-independent cross section of DM and the nucleon (b). The lime (yellow) band corresponds to the $1 \sigma(3 \sigma)$ range of Planck's DM relic density measurement [40]. The orange and black lines represent the upper limits on the spin-independent cross section of DM and the nucleon from the PandaX-II experiment [41] and XENON1T [42], respectively.

and direct searches. The allowed points in the parameter space are selected within $3 \sigma$ of Planck's DM relic density measurement [40] and below the upper limits of the DM direct detection searches from PandaX-II [41] and XENON1T [42]. Under these combined constraints of $(\mathrm{VS}+\mathrm{PU}+\mathrm{HP}+\mathrm{DM})$, the parameter space of the model is further reduced. For example, we now have that $-1 \leq$ $\lambda_{h_{1} h_{1} h_{1}} \leq 1.3$ and $\operatorname{BR}\left(h_{2} \rightarrow h_{1} h_{1}\right)$ is less than about $80 \%$. The negative value of $\lambda_{h_{1} h_{1} h_{1}}$ provides an enhancement of the production cross section because constructive interference occurs between the box and triangle diagrams. Overall, the production cross section of $h_{1}$ pairs is about 1 order of magnitude lower compared to the result before imposing the DM constraints.

\section{MONTE CARLO SIMULATIONS}

To proceed with our analysis, we pick seven benchmark points from the final allowed parameter space satisfying the $(\mathrm{VS}+\mathrm{PU}+\mathrm{HP}+\mathrm{DM})$ constraints, in which the mass of the heavier scalar $h_{2}$ varies from 300 to $900 \mathrm{GeV}$ in increments of $100 \mathrm{GeV}$, and label these benchmark points as A, B, C, D, E, F, and G respectively. In Table II, we show the fundamental parameters of the scalar potential, derived couplings, mass spectra of the scalars, and the signal strength for Higgs boson pair production at each benchmark point. Benchmark point A allows for a large branching ratio of $h_{2} \rightarrow h_{1} h_{1}$, which will allow us to study the enhancement effects of a heavy scalar resonance on the production cross section (see Table III), while for benchmark point $\mathrm{D}$ we have negative values for $\lambda_{h_{1} h_{1} h_{1}}$ which can lead to constructive interference between the box and triangle diagrams. For benchmark points E, F, and G, the production cross sections are about twice the SM value. One can see that both the magnitudes and signs of the Yukawa couplings between SM quarks and the $h_{1}$ Higgs boson are close to the SM values for all benchmark points except benchmark point $\mathrm{D}$, which has the opposite sign. In Table III, we show the branching ratios of $h_{2}$ decays into all two-body final states for our benchmark points. We observe that the heavy scalar $h_{2}$ mainly decays into the SM-like Higgs boson $h_{1}$ pair, $W / Z$ boson pairs, and to a lesser extent a top-quark pair. The branching ratio for $h_{2}$ decaying into a bottom quark pair is vanishingly small.

In order to perform detailed simulations at the LHC, we first implement the G2HDM model using FeynRules [43] and pass the Universal Feynrules Output model files to MADGRAPH5 AMC@NLO [44] to generate the $h_{1}$ pair production events to next-to-leading order in QCD in the heavy top-quark mass limit. The Higgs boson decay is done with MADSpin [45], and we focus on two decay modes: $h_{1} \rightarrow b \bar{b}$ and $h_{1} \rightarrow \gamma \gamma$. Finally, Pythia8 [46] is used for parton showering and hadronization, while DELPHES3 [47] (with the ATLAS setting) is used as the fast detector simulation.

In the next two subsections, we concentrate on the $\gamma \gamma b \bar{b}$ and $b \bar{b} b \bar{b}$ final states. According to the current Higgs boson pair production searches at the LHC, the $\gamma \gamma b \bar{b}$ final-state channel is a good search channel for the lower-mass regime of the heavy scalar mediator [48,49], while the $b \bar{b} b \bar{b}$ search channel has better sensitivity for the heavier-mass regime of the heavy scalar mediator [50,51]. Thus, we use our benchmark points A, B, C, and D to study the $\gamma \gamma b \bar{b}$ finalstate channel, while the benchmark points $\mathrm{E}, \mathrm{F}$, and $\mathrm{G}$ are used for the $b \bar{b} b \bar{b}$ final-state channel. 


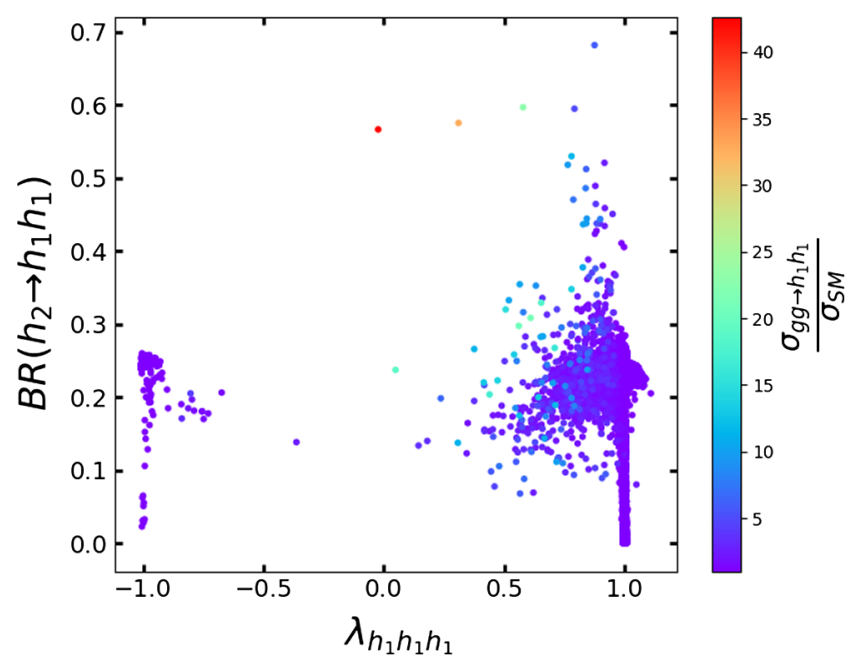

(a)

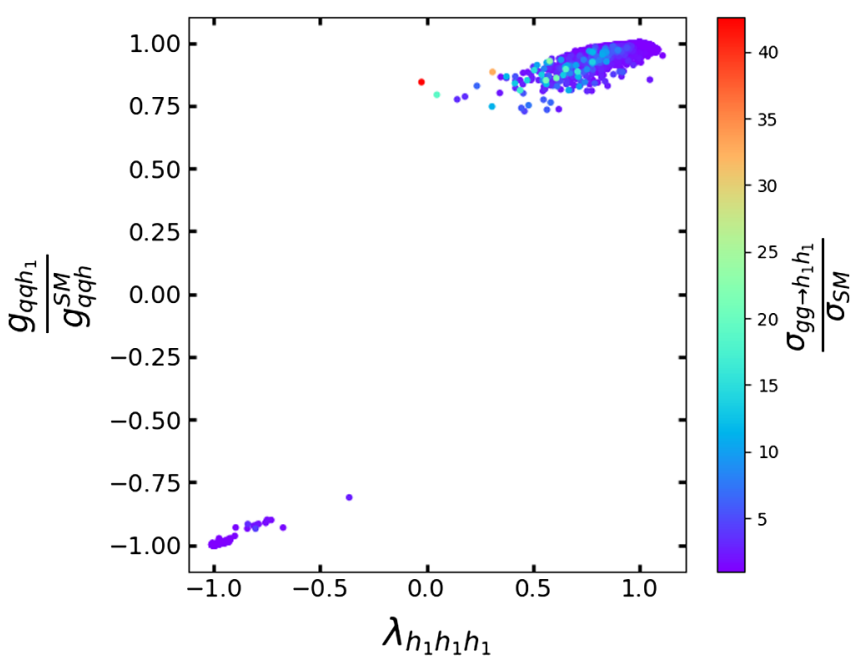

(c)

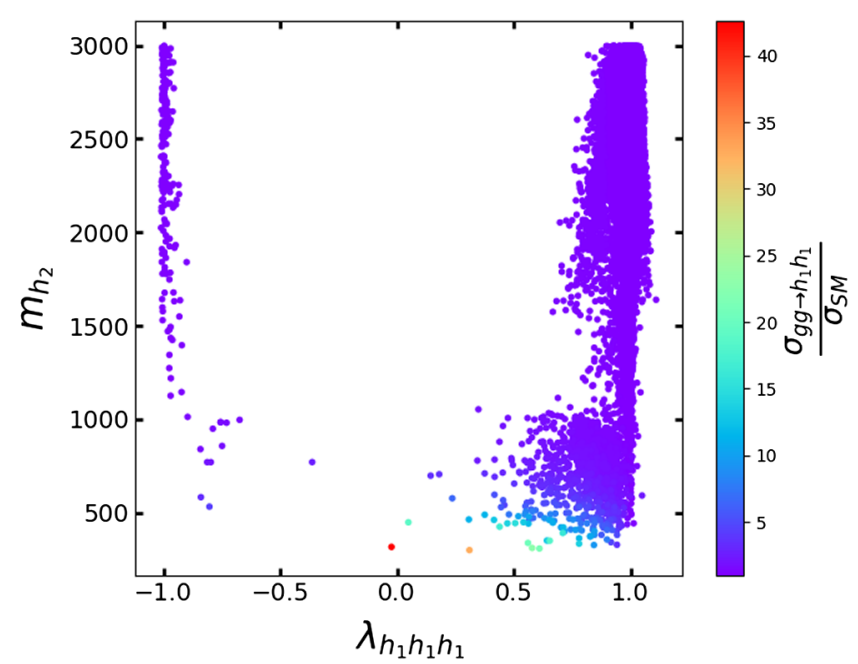

(b)

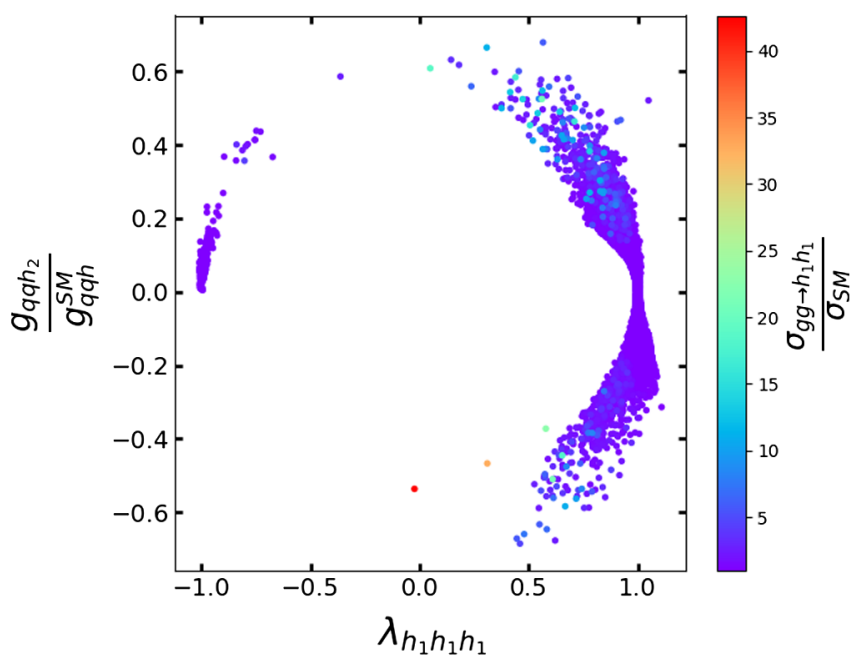

(d)

FIG. 5. Same as Fig. 3 but after taking into account the experimental constraints from Planck's DM relic density measurements [40] and direct searches from th ePandaX-II experiment [41] and XENON1T [42].

\section{A. The $\gamma \gamma b \bar{b}$ final-state channel}

In this section, we investigate various kinematic distributions of the $\gamma \gamma b \bar{b}$ final state. We select the four benchmark points $\mathrm{A}, \mathrm{B}, \mathrm{C}$, and $\mathrm{D}$ at which the mass of the heavy scalar $h_{2}$ is $300,400,500$, and $600 \mathrm{GeV}$ respectively, to study this channel. Here, we follow the cuts used in the ATLAS experiment for the $\gamma \gamma b \bar{b}$ channel analysis [48], which we summarize as follows:

(1) First, we isolate the two photons with opening angle $\Delta R=0.2$.

(2) Next, using the anti- $k_{T}$ algorithm jets are reconstructed with cone radius $R=0.4$ and required to have a pseudorapidity $|\eta|<2.5$ and transverse momentum $p_{T}>25 \mathrm{GeV}$.
(3) We require at least two photons and exactly two jets with $b$-tagging efficiency set at the default value of MADGRAPH5.

(4) The leading $b$ jet must have $p_{T}>55 \mathrm{GeV}$, while the subleading $b$ jet is required to have $p_{T}>35 \mathrm{GeV}$.

(5) Furthermore, the diphoton invariant mass $m_{\gamma \gamma}$ is required to lie between 105 and $160 \mathrm{GeV}$ and the $b$-jet pair invariant mass $m_{b \bar{b}}$ is required to lie between 95 and $135 \mathrm{GeV}$.

In Fig. 6, we present various normalized kinematic distributions of the $\gamma \gamma b \bar{b}$ channel for (a) the invariant mass of $\gamma \gamma b \bar{b}$, (b) the opening angle $\Delta R_{\gamma \gamma}$ of the two photons, and c) the opening angle $\Delta R_{b b}$ of the two $b$ jets, in both the SM and G2HDM with $m_{h_{2}}=300,400,500$, and $600 \mathrm{GeV}$ at 
TABLE II. Seven benchmark points allowed by the combined (VS + PU + HP + DM) constraints.

\begin{tabular}{|c|c|c|c|c|c|c|c|}
\hline Benchmark point & A & B & $\mathrm{C}$ & $\mathrm{D}$ & $\mathrm{E}$ & $\mathrm{F}$ & $\mathrm{G}$ \\
\hline$m_{h_{2}}(\mathrm{GeV})$ & 300 & 400 & 500 & 600 & 700 & 800 & 900 \\
\hline$m_{h_{3}}(\mathrm{TeV})$ & 141.35 & 68.23 & 93.59 & 87.86 & 88.35 & 69.67 & 92.71 \\
\hline$m_{D}(\mathrm{GeV})$ & 1051.7 & 814.6 & 905.4 & 487.2 & 883.3 & 880.4 & 767.1 \\
\hline$m_{\tilde{\Delta}}(\mathrm{TeV})$ & 71.20 & 84.42 & 48.26 & 109.34 & 44.38 & 65.18 & 72.86 \\
\hline$m_{H^{ \pm}}(\mathrm{TeV})$ & 71.21 & 84.41 & 48.26 & 109.34 & 44.39 & 65.18 & 72.86 \\
\hline$\lambda_{H}$ & 0.36 & 1.24 & 0.53 & 1.15 & 0.66 & 0.99 & 1.72 \\
\hline$\lambda_{\Phi}$ & 3.67 & 1.45 & 2.03 & 0.89 & 1.44 & 1.57 & 2.57 \\
\hline$\lambda_{\Delta}$ & 0.22 & 0.13 & 1.60 & 0.14 & 0.67 & 0.25 & 0.38 \\
\hline$\lambda_{H}^{\prime}$ & -19.8 & -2.47 & -3.77 & -3.51 & -17.80 & -3.65 & -2.75 \\
\hline$\lambda_{H \Phi}$ & -1.22 & -2.24 & -0.31 & -1.45 & 0.003 & -0.38 & -1.85 \\
\hline$\lambda_{H \Delta}$ & 0.054 & -0.36 & -0.17 & -0.15 & -0.316 & 0.15 & -0.99 \\
\hline$\lambda_{\Phi \Delta}$ & 2.12 & 0.95 & 3.63 & 0.08 & 2.08 & 1.44 & 1.97 \\
\hline$\lambda_{H \Phi}^{\prime}$ & 3.73 & 9.73 & 2.16 & 5.52 & 1.46 & 5.49 & 6.35 \\
\hline$v_{\Delta}(\mathrm{TeV})$ & 2.41 & 3.08 & 3.27 & 0.43 & 1.52 & 1.21 & 2.85 \\
\hline$v_{\Phi}(\mathrm{TeV})$ & 52.11 & 40.05 & 46.39 & 65.79 & 51.91 & 39.32 & 40.88 \\
\hline$M_{H \Delta}(\mathrm{GeV})$ & 2209 & 2776 & 1465 & 897 & -2293 & 2656 & 956.7 \\
\hline$M_{\Phi \Delta}(\mathrm{GeV})$ & 3.85 & 4.88 & 4.85 & 0.08 & 1.80 & 2.31 & 3.90 \\
\hline$\lambda_{h_{1} h_{1} h_{1}}$ & 0.31 & 0.71 & 0.55 & -0.84 & 0.84 & 0.75 & 0.58 \\
\hline$\lambda_{h_{2} h_{1} h_{1}}$ & -1.90 & 1.65 & 2.61 & 2.46 & -3.32 & 5.04 & 6.54 \\
\hline$\kappa_{q q h_{1}}$ & 0.88 & 0.89 & 0.90 & -0.92 & 0.93 & 0.91 & 0.89 \\
\hline$\kappa_{q q h_{2}}$ & -0.47 & 0.46 & 0.44 & 0.40 & -0.37 & 0.41 & 0.44 \\
\hline$\kappa_{q^{H} q^{H} h_{1}}$ & $-2.6 \times 10^{-5}$ & $-4.6 \times 10^{-5}$ & $-1.4 \times 10^{-4}$ & $-1 \times 10^{-5}$ & $3.7 \times 10^{-5}$ & $-3.1 \times 10^{-5}$ & $5.8 \times 10^{-5}$ \\
\hline$\kappa_{q^{H} q^{H} h_{2}}$ & $-5.7 \times 10^{-5}$ & $1.5 \times 10^{-4}$ & $3.0 \times 10^{-4}$ & $5.6 \times 10^{-6}$ & $9.2 \times 10^{-5}$ & $8.2 \times 10^{-5}$ & $1.5 \times 10^{-4}$ \\
\hline$\frac{\sigma\left(g g \rightarrow h_{1} h_{1}\right)}{\sigma_{\mathrm{SM}}}$ & 32.4 & 16.1 & 7.9 & 3.3 & 2.1 & 2.1 & 2.0 \\
\hline
\end{tabular}

the LHC with $\sqrt{s}=13 \mathrm{TeV}$. In Fig. 6(a), it is obvious that the normalized invariant mass distributions for $\gamma \gamma b \bar{b}$, divided by the bin size $1200 / 120=10 \mathrm{GeV}$, peak at the corresponding mass of the heavy scalar $h_{2}$, while the peak around $400 \mathrm{GeV}$ is for the SM. We will discuss this SM peak further in the next subsection. The peaks decrease as the mass of $h_{2}$ increases, due to the fact that when the mass of $h_{2}$ increases the nonresonant process becomes more relevant to the total production cross section. One can also observe that the normalized distributions of the opening angles of the two photons and of the pair of $b$ jets divided by the bin size $2 \pi / 100$ in Figs. 6(b) and 6(c), respectively, become narrower when the mass of $h_{2}$ increases. This is

TABLE III. Branching ratios of the two-body decays of $h_{2}$ for the seven benchmark points.

\begin{tabular}{lccccccc}
\hline \hline Benchmark point & $\mathrm{A}$ & $\mathrm{B}$ & $\mathrm{C}$ & $\mathrm{D}$ & $\mathrm{E}$ & $\mathrm{F}$ & $\mathrm{G}$ \\
\hline$h_{2} \rightarrow h_{1} h_{1}$ & 0.564 & 0.261 & 0.245 & 0.167 & 0.175 & 0.198 & 0.181 \\
$h_{2} \rightarrow W^{+} W^{-}$ & 0.301 & 0.454 & 0.420 & 0.465 & 0.471 & 0.469 & 0.489 \\
$h_{2} \rightarrow Z Z$ & 0.134 & 0.211 & 0.201 & 0.224 & 0.230 & 0.230 & 0.241 \\
$h_{2} \rightarrow t \bar{t}$ & 0 & 0.073 & 0.133 & 0.143 & 0.122 & 0.102 & 0.088 \\
$h_{2} \rightarrow b \bar{b}$ & $\sim 0$ & $\sim 0$ & $\sim 0$ & $\sim 0$ & $\sim 0$ & $\sim 0$ & $\sim 0$ \\
\hline \hline
\end{tabular}

expected because when the parent decaying particle $h_{2}$ becomes heavier, the two daughter $h_{1}$ Higgs bosons will be more boosted, implying that the opening angles $\Delta R_{\gamma \gamma}$ and $\Delta R_{b b}$ would be smaller.

As mentioned above, the invariant mass distribution of the Higgs boson pair peaks at about $400 \mathrm{GeV}$ in the case of the SM, even though there is no new resonance. A natural question arises: could we tell whether there exists a new scalar resonance $S$ if the observed total cross section does not deviate much from the SM prediction? To answer this question, we will temporarily leave the G2HDM and do some shape comparisons in a hypothetical scenario defined below.

In Figs. 7(a) and 7(b), we perform a standard deviation $\chi^{2}$ test for the event distributions in $M_{\gamma \gamma b b}$ and $\Delta R_{\gamma \gamma}$, respectively, at the high-luminosity LHC for the SM and a generic scalar resonance $S$ with a mass of $400 \mathrm{GeV}$ and a total production cross section that matches the SM prediction. The error is naively taken as the square root of the pseudodata bin content. It turns out that at the $13 \mathrm{TeV}$ LHC with $\mathcal{L}_{\text {int }}=3000 \mathrm{fb}^{-1}$, we cannot tell the difference between a new physics signal of the heavy scalar resonance with a mass of $400 \mathrm{GeV}$ and the SM predictions in Higgs boson pair production. In particular, the deviations from the 


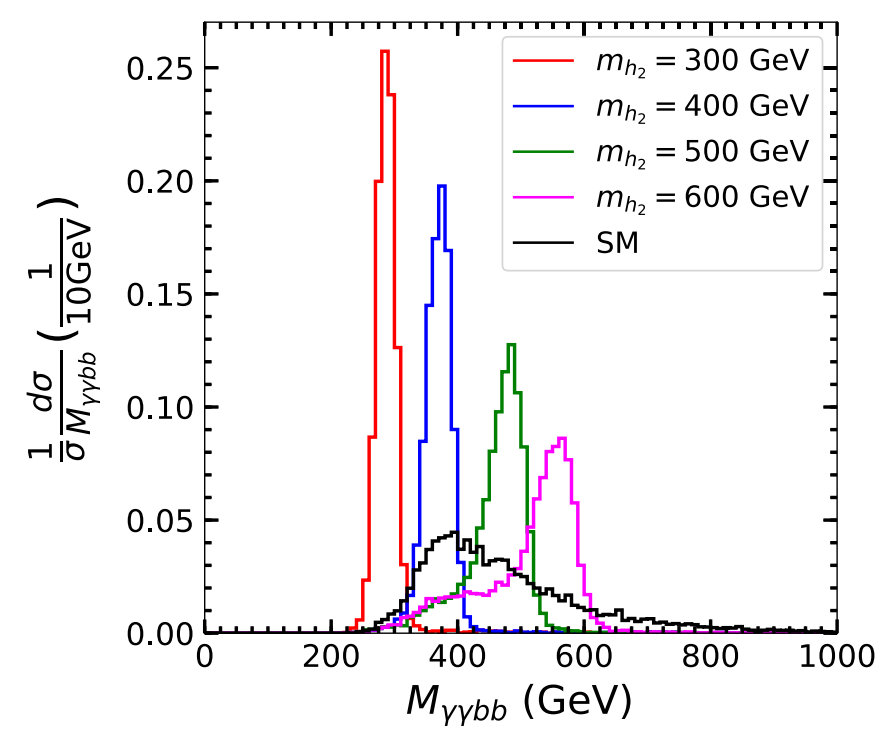

(a)

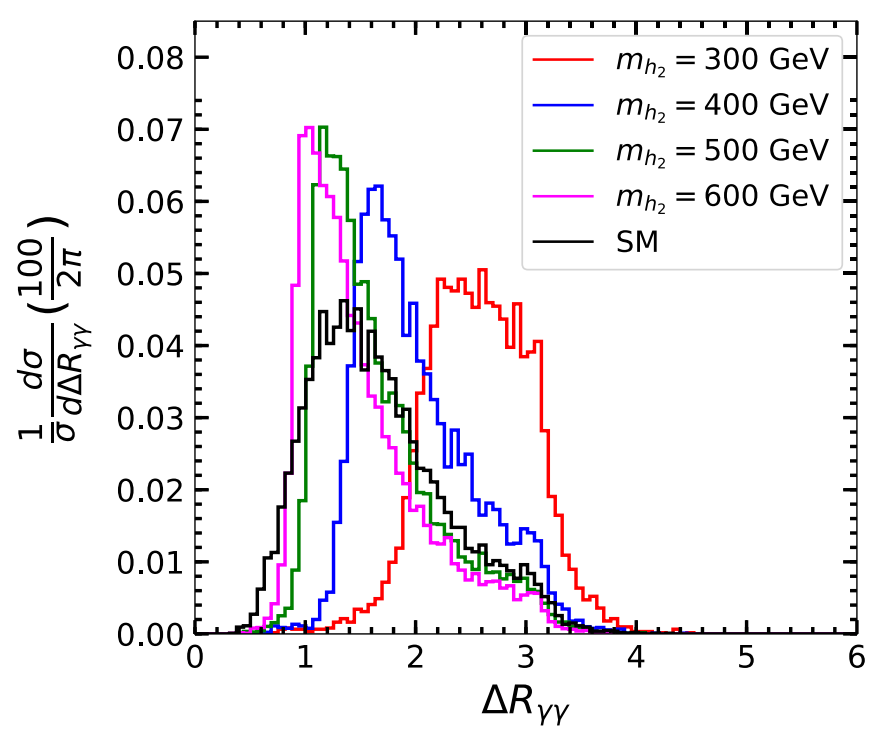

(b)

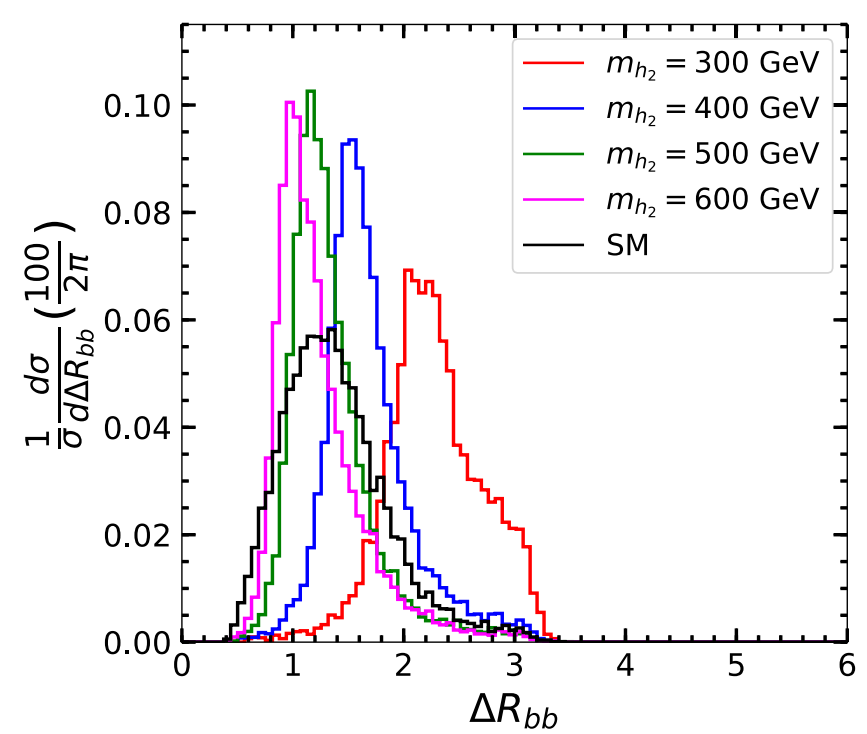

(c)

FIG. 6. Normalized kinematic distributions of the $\gamma \gamma b \bar{b}$ channel for (a) the invariant mass $M_{\gamma \gamma b b}$ of $\gamma \gamma b \bar{b}$, (b) the opening angle $\Delta R_{\gamma \gamma}$ of the two photons, and (c) the opening angle $\Delta R_{b b}$ of the two $b$ jets, in both the SM and G2HDM with $m_{h_{2}}=300,400,500$, and $600 \mathrm{GeV}$ at the $\sqrt{s}=13 \mathrm{TeV}$ LHC.

SM predictions are about $1.31 \sigma$ in the case of the $M_{\gamma \gamma b b}$ kinematic distribution and only $0.03 \sigma$ in the case of the $\Delta R_{\gamma \gamma}$ distribution.

However, the situation can be improved at a future hadron collider. For illustration, we present the corresponding integrated luminosity versus standard deviation $\chi^{2}$ test for the generic $400 \mathrm{GeV}$ scalar resonance and the SM one at a $100 \mathrm{TeV}$ LHC. The result shown in Fig. 8 indicates that with an integrated luminosity $\mathcal{L}_{\text {int }} \sim 500 \mathrm{fb}^{-1}$ at a $100 \mathrm{TeV}$ LHC, the signal for the $400 \mathrm{GeV}$ scalar resonance can be distinguished from the SM at the $3 \sigma$ level.

\section{B. The $b \bar{b} b \bar{b}$ final-state channel}

We now return to the G2HDM and select the benchmark points E, F, and $\mathrm{G}$ at which $m_{h_{2}}=700,800$, and $900 \mathrm{GeV}$, respectively, to study the kinematic distributions of the $b \bar{b} b \bar{b}$ final-state channel. In this case, we follow the event selections used in the ATLAS resolved analysis for the $b \bar{b} b \bar{b}$ final-state channel [50], which we summarize below:

(1) To be specific, the events are required to contain at least four $b$ jets with $p_{T}>30 \mathrm{GeV}$ and $|\eta|<2.5$. 


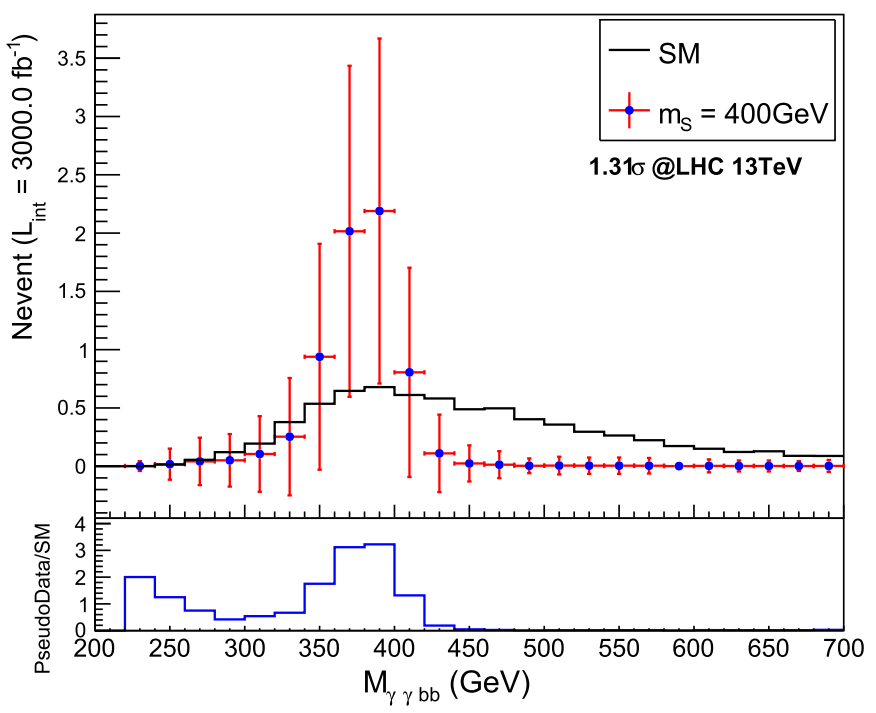

(a)

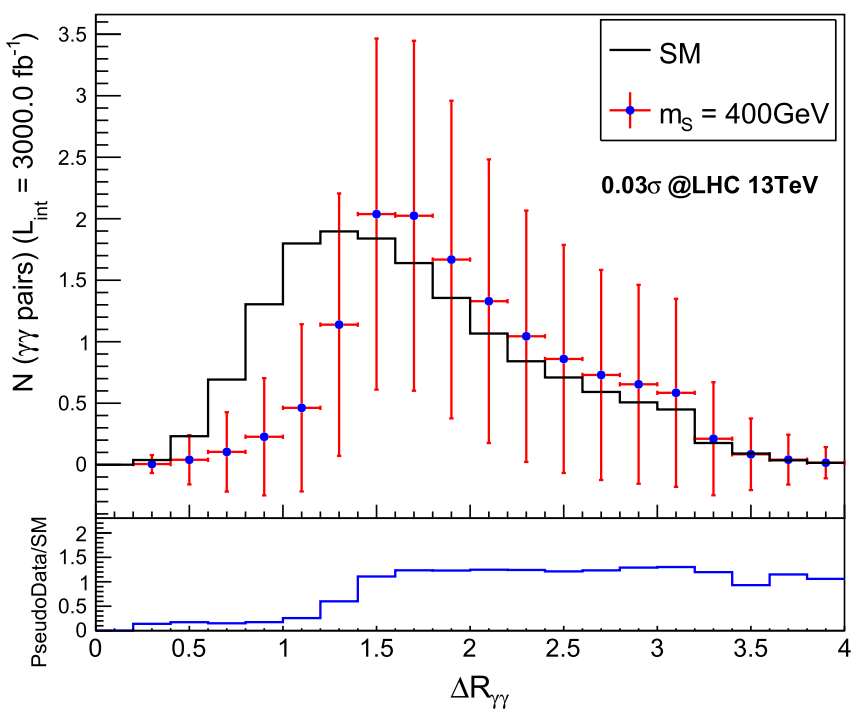

(b)

FIG. 7. Standard deviation $\chi^{2}$ test for pair production of the SM Higgs via a generic $400 \mathrm{GeV}$ scalar resonance $S$ versus the SM prediction at the $13 \mathrm{TeV}$ LHC with an integrated luminosity of $\mathcal{L}_{\text {int }}=3000 \mathrm{fb}^{-1}$.

(2) Furthermore, we pair up these four $b$ jets to reconstruct two $125 \mathrm{GeV}$ Higgs boson candidates and then impose additional mass-dependent cuts for these two Higgs boson candidates as follows:

$$
\begin{gathered}
\left.\begin{array}{c}
\frac{360}{m_{4 j} / \mathrm{GeV}}-0.5<\Delta R_{j j}^{\text {lead }}<\frac{653}{m_{4 j} / \mathrm{GeV}}+0.475 \\
\frac{235}{m_{4 j} / \mathrm{GeV}}<\Delta R_{j j}^{\text {subl }}<\frac{875}{m_{4 j} / \mathrm{GeV}}+0.35
\end{array}\right\} \text { if } m_{4 j}<1250 \mathrm{GeV}, \\
\left.\begin{array}{c}
0<\Delta R_{j j}^{\text {lead }}<1 \\
0<\Delta R_{j j}^{\text {subl }}<1
\end{array}\right\} \text { if } m_{4 j}>1250 \mathrm{GeV},
\end{gathered}
$$

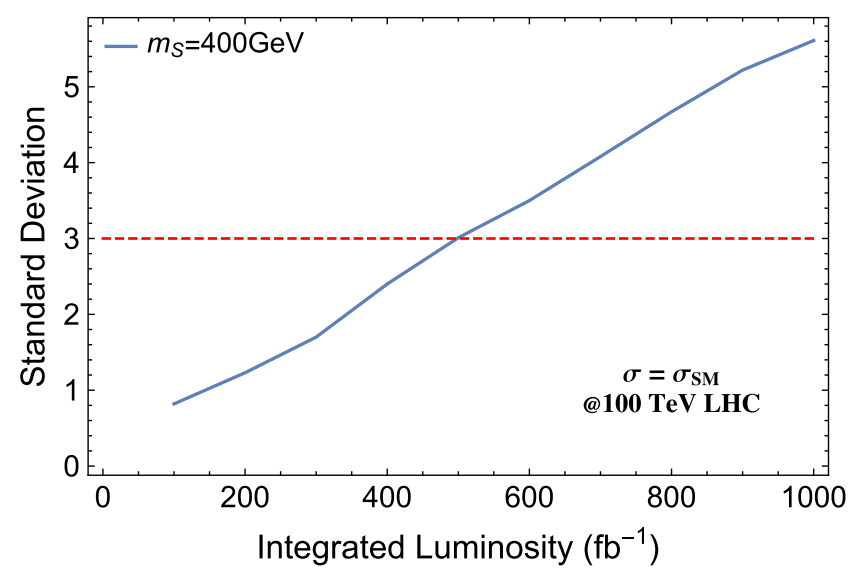

FIG. 8. The integrated luminosity versus standard deviation $\chi^{2}$ test for pair production of the SM Higgs via a generic $400 \mathrm{GeV}$ Higgs resonance $S$ versus the SM prediction at the $100 \mathrm{TeV}$ LHC. $\sigma=\sigma_{\mathrm{SM}}$ means that we set the cross section for the process $g g \rightarrow$ $S(400) \rightarrow h_{1} h_{1} \rightarrow \gamma \gamma \bar{b} b$ via the generic $400 \mathrm{GeV}$ scalar resonance to be the same as the SM prediction. where $\Delta R_{j j}^{\text {lead/subl }}$ is the opening angle of the two jets that the leading/subleading Higgs boson candidate decays into. Here, the leading Higgs boson candidate refers to the reconstructed Higgs boson that has the larger scalar sum of jet $p_{T}$.

(3) Then, an algorithm is applied to select the best pairing of $b$ jets into two Higgs boson candidates as follows. A distance $D_{h_{1}, h_{1}}$ [50] is defined as

$$
\begin{aligned}
D_{h_{1}, h_{1}}= & \sqrt{\left(m_{2 j}^{\text {lead }}\right)^{2}+\left(m_{2 j}^{\text {subl }}\right)^{2}} \\
& \times\left|\sin \left(\tan ^{-1}\left(\frac{m_{2 j}^{\text {subl }}}{m_{2 j}^{\text {lead }}}\right)-\tan ^{-1}\left(\frac{115}{120}\right)\right)\right|,
\end{aligned}
$$

which is the distance of the reconstructed pairing's point $\left(m_{2 j}^{\text {lead }}, m_{2 j}^{\text {subl }}\right)$ to the line connecting the two points $(0 \mathrm{GeV}, 0 \mathrm{GeV})$ and $(120 \mathrm{GeV}, 115 \mathrm{GeV})$ in 


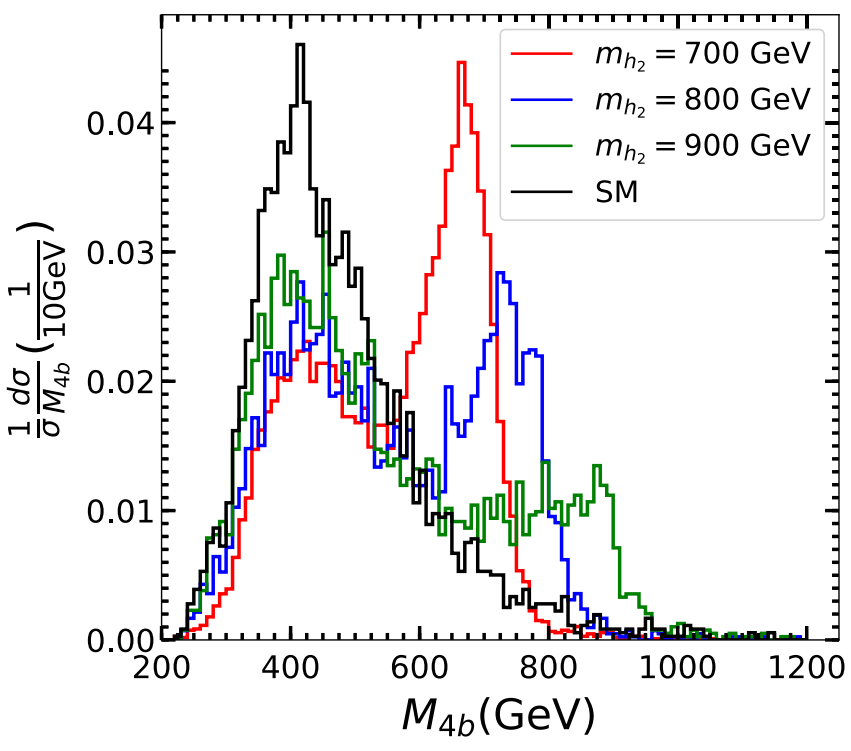

(a)

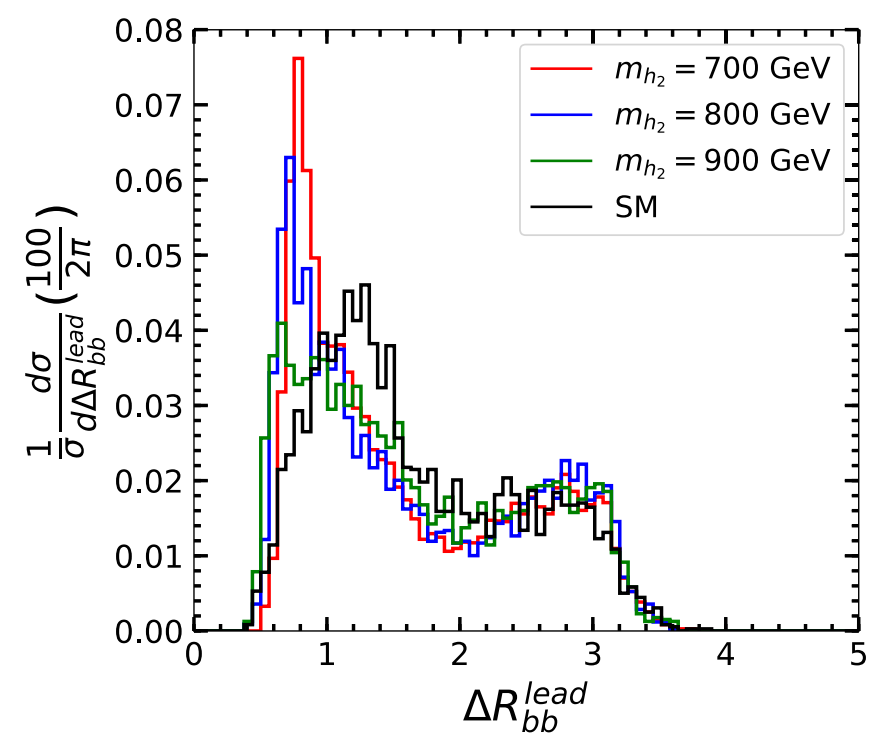

(b)

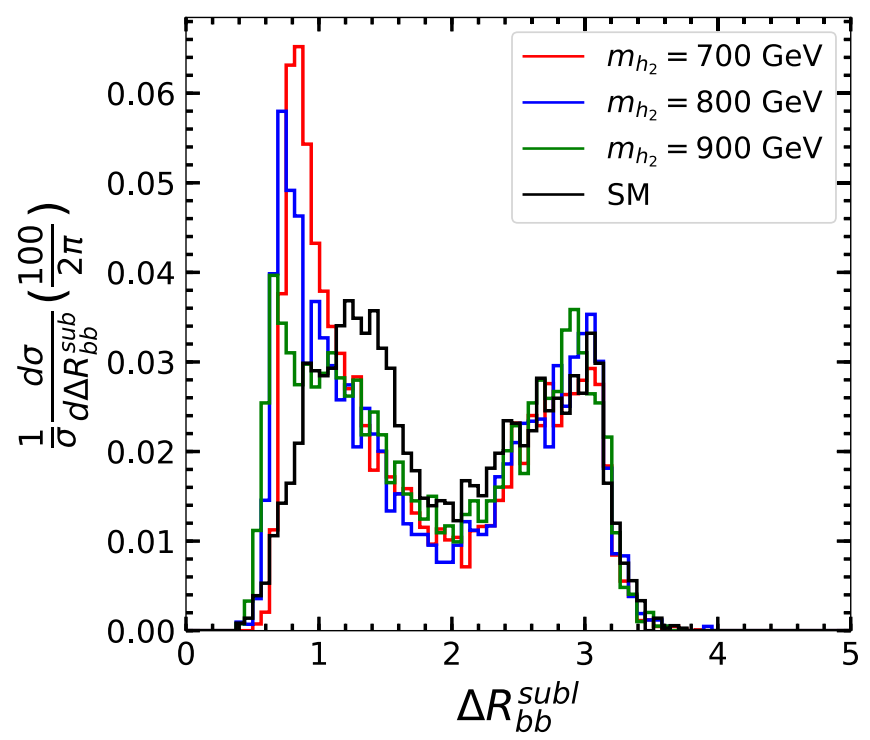

(c)

FIG. 9. Normalized kinematic distributions of the $b \bar{b} b \bar{b}$ channel for (a) the invariant mass $M_{4 b}$ of $b \bar{b} b \bar{b}$, (b) the opening angle $\Delta R_{b b}^{\text {lead }}$ of the two $b$ jets associated with the leading Higgs boson candidate, and (c) the opening angle $\Delta R_{b b}^{\text {subl }}$ of the two $b$ jets associated with the subleading Higgs boson candidate with $m_{h_{2}}=700,800$, and $900 \mathrm{GeV}$ for the ATLAS detectors at $\sqrt{s}=13 \mathrm{TeV}$. The results for the SM are also shown for comparison.

the $m_{2 j}^{\text {lead }}-m_{2 j}^{\text {subl }}$ plane. Here, $m_{2 j}^{\text {lead } / \text { subl }}$ is the mass of the leading/subleading Higgs boson candidate and the values of 120 and $115 \mathrm{GeV}$ are basically the centers of the signal regions for $m_{2 j}^{\text {lead }}$ and $m_{2 j}^{\text {subl }}$, respectively. Among the Higgs boson candidates, the pair that has the minimum distance $D_{h_{1}, h_{1}}$ is defined to be the two Higgs bosons.

(4) In addition, the two Higgs boson candidates are required to have transverse momenta $p_{T}^{\text {lead }}$ and $p_{T}^{\text {subl }}$, opening angle $\Delta R\left(h_{1}, h_{1}\right)$, and pseudorapidity difference $\left|\Delta \eta_{h_{1} h_{1}}\right|$ that satisfy the following massdependent cuts:

$$
\begin{aligned}
p_{T}^{\text {lead }} & >0.5 m_{4 j}-90 \mathrm{GeV}, \\
p_{T}^{\text {subl }} & >0.33 m_{4 j}-70 \mathrm{GeV}, \\
\Delta R\left(h_{1}, h_{1}\right) & >1.5,
\end{aligned}
$$


and

$$
\left|\Delta \eta_{h_{1} h_{1}}\right|< \begin{cases}1.1 & \text { if } m_{4 j}<850 \mathrm{GeV}, \\ 2 \times 10^{-3}\left(m_{4 j} / \mathrm{GeV}\right)-0.6 & \text { if } m_{4 j}>850 \mathrm{GeV} .\end{cases}
$$

(5) Finally, the mass of the Higgs boson candidates must lie within the signal region $X_{h_{1}, h_{1}}$ defined by [50]

$$
X_{h_{1}, h_{1}}=\sqrt{\left(\frac{m_{2 j}^{\text {lead }}-120 \mathrm{GeV}}{0.1 m_{2 j}^{\text {lead }}}\right)^{2}+\left(\frac{m_{2 j}^{\text {subl }}-115 \mathrm{GeV}}{0.1 m_{2 j}^{\text {subl }}}\right)^{2}}<1.6 .
$$

In Fig. 9, we present various normalized kinematic distributions of the $b \bar{b} b \bar{b}$ channel for (a) the invariant mass $M_{4 b}$ of $b \bar{b} b \bar{b}$, (b) the opening angle $\Delta R_{b b}^{\text {lead }}$ of the two $b$ jets associated with the leading Higgs boson candidate, and (c) the opening angle $\Delta R_{b b}^{\text {subl }}$ of the two $b$ jets associated with the subleading Higgs boson candidate, with $m_{h_{2}}=700,800$, and $900 \mathrm{GeV}$ for the ATLAS detectors at $\sqrt{s}=13 \mathrm{TeV}$. For comparison, the results for the SM are also shown. We note that the three benchmark points (E, F, and G) selected to study this $b \bar{b} b \bar{b}$ final-state channel have the same production cross sections and are about twice the SM value (see Table II).

In Fig. 9(a), for the SM one can observe that the nonresonant contributions from both the box and triangle diagrams in the invariant mass distribution for $b \bar{b} b \bar{b}$ peak around $400 \mathrm{GeV}$. Recall that in the SM the box diagram is dominant while the triangle diagram interferes destructively with the box. On the other hand, for the benchmark points E, F, and G in the G2HDM with $m_{h_{2}}=700,800$, and $900 \mathrm{GeV}$, respectively, there are two peaks: besides the original ones around $400 \mathrm{GeV}$, there are peaks located around 700,800 , and $900 \mathrm{GeV}$, presumably due to the resonant contributions from on-shell $h_{2}$ decay in the triangle diagram. Thus, this invariant mass distribution in $M_{4 b}$ is useful to distinguish between these benchmark points in the G2HDM and the SM.

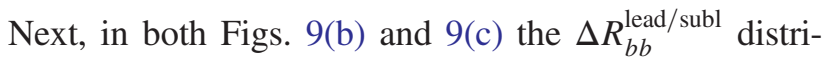
butions tend to separate into two peaks-one at $\Delta R_{b b}^{\text {lead/subl }} \approx 1$ and another at $\Delta R_{b b}^{\text {lead/subl }} \approx 3$-in both the G2HDM and SM. This behavior is somewhat expected in the G2HDM because of the different contributions from nonresonant and resonant processes to the total production cross section, keeping in mind that both the triangle and box diagrams in the SM are nonresonant. In particular, the peak at about 1 mainly comes from the $h_{2}$ resonance production in the G2HDM, while the box diagram is important in the case of the SM. On the other hand, the peak at about 3 represents the main contribution of the nonresonance triangle diagram for both the G2HDM and the SM. Therefore, one may be able to use the opening angle $\Delta R_{b b}^{\text {lead/subl }}$ to separate the nonresonant and resonant contributions in the G2HDM. While the distributions look rather similar at $\Delta R_{b b}^{\text {lead/subl }} \approx 3$ in both the G2HDM and $\mathrm{SM}$, they begin to differ at about 1 in both Figs. 9(b) and 9(c). Finally, we note that the $\Delta R_{b b}^{\text {lead }}$ distribution in Fig. 9(b) is more likely to have a peak located at about 1 due to its more energetic parent $h_{2}$ in the triangle diagram in the G2HDM.

\section{CONCLUSION}

Exploring Higgs boson pair production at the LHC is an important way to probe for the Higgs boson self-coupling in the SM, in particular the trilinear term in the Higgs potential, which is a unique trace of electroweak symmetry breaking via the Higgs mechanism. It is also an interesting channel to search for new physics beyond the SM, especially for models that involve a new Higgs resonance decaying into a Higgs boson pair. We have studied this process in the G2HDM, a model which promotes the discrete $Z_{2}$ symmetry which ensures the stability of DM in the IHDM to a local gauge symmetry, by embedding the two Higgs doublets into the fundamental representation of the new $S U(2)_{H}$ gauge group. One interesting feature in the G2HDM is that the $125 \mathrm{GeV} \mathrm{SM-like} \mathrm{Higgs} \mathrm{boson} \mathrm{is} \mathrm{in}$ general a linear combination of the $S U(2)_{L}$ doublet and the $S U(2)_{H}$ doublet and triplet scalars, which may lead to deviations in the interactions of the Higgs boson with not only other particles but also itself, as compared with the SM couplings. In particular, the Higgs boson self-coupling could become negative, which will result in constructive interference between the box and triangle Feynman diagrams. Moreover, the existence of the heavy scalar bosons in the G2HDM, which will decay into two $125 \mathrm{GeV}$ Higgs bosons, can significantly enhance the production cross section of Higgs boson pairs at the LHC.

We have taken into account the theoretical (VS + PU) and experimental (HP) constraints imposed upon the parameter space of the model. The DM constraints, including the DM relic density and direct detection searches, have also been imposed. We found that the Higgs boson trilinear coupling is stringently constrained by the DM relic density and direct searches. In particular, $\lambda_{h_{1} h_{1} h_{1}}$, the trilinear self-coupling of the $125 \mathrm{GeV}$ Higgs in 
the G2HDM normalized to its SM value (which could vary in the range $[-29,32]$ before the DM constraints are applied) now varies in the range $[-1,1.3]$. This results in a reduction of the double Higgs boson production cross section in the G2HDM from about 2 orders of magnitude enhancement before applying the DM constraints to just about 1 order of magnitude above the SM value. This range of $\lambda_{h_{1} h_{1} h_{1}}$ could be probed by the high-luminosity LHC (HL-LHC) with $3 \mathrm{ab}^{-1}$.

We have performed detailed simulations for the two final states of $\gamma \gamma b \bar{b}$ and $b \bar{b} b \bar{b}$ from the decays of the Higgs boson pair by focusing on a few benchmark points of the parameter space in the G2HDM. All of these benchmark points satisfy the (VS $+\mathrm{PU}+\mathrm{HP}+\mathrm{DM})$ constraints. The G2HDM demonstrates a representative case in which the kinematic distributions of the $\gamma \gamma b \bar{b}$ and $b \bar{b} b \bar{b}$ final-state channels are significantly altered from the SM predictions at the $\sqrt{s}=13 \mathrm{TeV}$ LHC. We also presented a standard deviation $\chi^{2}$ test for the signals of Higgs pair production via a generic scalar resonance. It turns out that it is impossible to distinguish a $400 \mathrm{GeV}$ scalar resonant signal from the $\mathrm{SM}$ at the HL-LHC running at $13 \mathrm{TeV}$, if the total cross section of such a resonance is in close proximity to the one in the SM. However, at future machines with higher centerof-mass energies and luminosities it may be possible to observe this signal from a new scalar resonance.

\section{ACKNOWLEDGMENTS}

We thank Dr. Raymundo Ramos and Dr. Yue-Lin Sming Tsai for useful discussions and acknowledge the support by the National Center for Theoretical Sciences (NCTS). This work was supported in part by the Ministry of Science and Technology (MoST) of Taiwan under Grants No. 1072119-M-001-033-, No. 105-2112-M-003-010-MY3 and No. 104-2112-M-001-001-MY3.
[1] See "The Standard Model At Fifty Years: A Celebratory Symposium" at http://artsci.case.edu/smat50/.

[2] M. Aaboud et al. (ATLAS Collaboration), Phys. Lett. B 786, 59 (2018).

[3] A. M. Sirunyan et al. (CMS Collaboration), Phys. Rev. Lett. 121, 121801 (2018).

[4] G. C. Branco, P. M. Ferreira, L. Lavoura, M. N. Rebelo, M. Sher, and J. P. Silva, Phys. Rep. 516, 1 (2012).

[5] N. G. Deshpande and E. Ma, Phys. Rev. D 18, 2574 (1978).

[6] E. Ma, Phys. Rev. D 73, 077301 (2006).

[7] R. Barbieri, L. J. Hall, and V. S. Rychkov, Phys. Rev. D 74, 015007 (2006).

[8] L. Lopez Honorez, E. Nezri, J. F. Oliver, and M. H. G. Tytgat, J. Cosmol. Astropart. Phys. 02 (2007) 028.

[9] A. Arhrib, Y. L. S. Tsai, Q. Yuan, and T. C. Yuan, J. Cosmol. Astropart. Phys. 06 (2014) 030.

[10] S. L. Glashow and S. Weinberg, Phys. Rev. D 15, 1958 (1977).

[11] W. C. Huang, Y. L. S. Tsai, and T. C. Yuan, J. High Energy Phys. 04 (2016) 019.

[12] P. Ko, Y. Omura, and C. Yu, J. High Energy Phys. 11 (2014) 054.

[13] P. Ko, Y. Omura, and C. Yu, Phys. Lett. B 717, 202 (2012).

[14] M. D. Campos, D. Cogollo, M. Lindner, T. Melo, F. S. Queiroz, and W. Rodejohann, J. High Energy Phys. 08 (2017) 092.

[15] W. C. Huang, Y. L. S. Tsai, and T. C. Yuan, Nucl. Phys. B909, 122 (2016).

[16] W. C. Huang, H. Ishida, C. T. Lu, Y. L. S. Tsai, and T. C. Yuan, Eur. Phys. J. C 78, 613 (2018).

[17] A. Arhrib, W. C. Huang, R. Ramos, Y. L. S. Tsai, and T. C. Yuan, Phys. Rev. D 98, 095006 (2018).

[18] A. Arhrib, R. Benbrik, C. H. Chen, R. Guedes, and R. Santos, J. High Energy Phys. 08 (2009) 035.
[19] G. Degrassi, P. P. Giardino, F. Maltoni, and D. Pagani, J. High Energy Phys. 12 (2016) 080.

[20] F. Maltoni, D. Pagani, A. Shivaji, and X. Zhao, Eur. Phys. J. C 77, 887 (2017).

[21] W. Yao, arXiv:1308.6302.

[22] V. Barger, L. L. Everett, C. B. Jackson, and G. Shaughnessy, Phys. Lett. B 728, 433 (2014).

[23] E. W. N. Glover and J. J. van der Bij, Nucl. Phys. B309, 282 (1988).

[24] S. Dawson, S. Dittmaier, and M. Spira, Phys. Rev. D 58, 115012 (1998).

[25] D. de Florian and J. Mazzitelli, Phys. Lett. B 724, 306 (2013).

[26] D. de Florian and J. Mazzitelli, Phys. Rev. Lett. 111, 201801 (2013).

[27] D. de Florian and J. Mazzitelli, J. High Energy Phys. 09 (2015) 053.

[28] D. de Florian, M. Grazzini, C. Hanga, S. Kallweit, J. M. Lindert, P. Maierhöfer, J. Mazzitelli, and D. Rathlev, J. High Energy Phys. 09 (2016) 151.

[29] S. Borowka, N. Greiner, G. Heinrich, S. P. Jones, M. Kerner, J. Schlenk, and T. Zirke, J. High Energy Phys. 10 (2016) 107.

[30] M. Spira, Prog. Part. Nucl. Phys. 95, 98 (2017).

[31] D. de Florian et al. (LHC Higgs Cross Section Working Group), arXiv:1610.07922.

[32] S. Borowka, N. Greiner, G. Heinrich, S. P. Jones, M. Kerner, J. Schlenk, U. Schubert, and T. Zirke, Phys. Rev. Lett. 117, 012001 (2016); 117, 079901(E) (2016).

[33] T. Plehn, M. Spira, and P. M. Zerwas, Nucl. Phys. B479, 46 (1996); B531, 655 (1998).

[34] (The ATLAS Collaboration), Report No. ATLAS-CONF2018-043. 
[35] M. Aaboud et al. (ATLAS Collaboration), Phys. Rev. D 96, 052004 (2017).

[36] M. Aaboud et al. (ATLAS Collaboration), Phys. Lett. B 761, 372 (2016).

[37] (The CMS Collaboration), Report No. CMS-PAS-EXO-16031.

[38] (The CMS Collaboration), Report No. CMS-PAS-EXO-16032 .

[39] G. Bélanger, F. Boudjema, A. Goudelis, A. Pukhov, and B. Zaldivar, Comput. Phys. Commun. 231, 173 (2018).

[40] N. Aghanim et al. (Planck Collaboration), arXiv:1807.06209.

[41] X. Cui et al. (PandaX-II Collaboration), Phys. Rev. Lett. 119, 181302 (2017).

[42] E. Aprile et al. (XENON Collaboration), Phys. Rev. Lett. 121, 111302 (2018).

[43] A. Alloul, N. D. Christensen, C. Degrande, C. Duhr, and B. Fuks, Comput. Phys. Commun. 185, 2250 (2014).
[44] J. Alwall, R. Frederix, S. Frixione, V. Hirschi, F. Maltoni, O. Mattelaer, H.-S. Shao, T. Stelzer, P. Torrielli, and M. Zaro, J. High Energy Phys. 07 (2014) 079.

[45] P. Artoisenet, R. Frederix, O. Mattelaer, and R. Rietkerk, J. High Energy Phys. 03 (2013) 015.

[46] T. Sjostrand, S. Mrenna, and P.Z. Skands, Comput. Phys. Commun. 178, 852 (2008).

[47] J. de Favereau, C. Delaere, P. Demin, A. Giammanco, V. Lemaître, A. Mertens, and M. Selvaggi (DELPHES 3 Collaboration), J. High Energy Phys. 02 (2014) 057.

[48] (The ATLAS Collaboration), Report No. ATLAS-CONF2016-004.

[49] (The CMS Collaboration), Report No. CMS-PAS-HIG-17008.

[50] (The ATLAS Collaboration), Report No. ATLAS-CONF2016-049.

[51] (The CMS Collaboration), Report No. CMS-PAS-HIG-16026. 